\title{
External leg corrections in the unitarity method
}

\section{Ruth Britto and Edoardo Mirabella}

Institut de Physique Théorique, CEA-Saclay,

F-91191, Gif-sur-Yvette cedex, France

E-mail: ruth.britto@cea.fr, edoardo.mirabella@cea.fr

ABSTRACT: Unitarity cuts diverge in the channel of a single massive external fermion. We propose an off-shell continuation of the momentum that allows a finite evaluation of the unitarity cuts. If the cut is taken with complete amplitudes on each side, our continuation and expansion around the on-shell configuration produces the finite contribution to the bubble coefficient. Finite parts in the expansion of the external leg counterterms must be included explicitly as well.

KeYwords: NLO Computations, QCD

ArXiv EPrint: 1109.5106 


\section{Contents}

1 Introduction 1

2 Description of the method 3

2.1 Bubbles from double cut 4

$\begin{array}{ll}2.2 & \text { Tadpole from single cut }\end{array}$

$\begin{array}{lll}2.3 & \text { Cancellation against the counterterm } & 7\end{array}$

$\begin{array}{llr}3 & \text { Examples } & 8\end{array}$

3.1 The process $H \rightarrow b \bar{b} \quad 8$

$\begin{array}{lll}3.2 & \text { The process } q \bar{q} \rightarrow t \bar{t} & 11\end{array}$

4 On-shell bubbles in the spinor-helicity formalism 12

$\begin{array}{ll}\text { 4.1 Contributions of the unrenormalized amplitude } & 13\end{array}$

$\begin{array}{ll}\text { 4.1.1 The cut in the } k_{1}^{2} \text { channel } & 14\end{array}$

$\begin{array}{ll}\text { 4.1.2 The cut in the } k_{2}^{2} \text { channel } & 16\end{array}$

$\begin{array}{lll}4.2 & \text { Contributions of the counterterm diagrams } & 16\end{array}$

$\begin{array}{lll}4.3 & \text { Total bubble coefficient } & 17\end{array}$

$\begin{array}{llr}5 & \text { Summary } & 18\end{array}$

$\begin{array}{ll}\text { A Master integrals, double and single cuts } & 19\end{array}$

B Massive spinors $\quad 20$

B.1 Some identities for massive spinors $\quad 21$

B.2 Tree-level formulas 21

\section{Introduction}

Precision computations beyond the leading order (LO) play a crucial role in the physical analyses performed at the LHC. Several new-physics signals, as well as many background processes, involve multi-particle final states. At LO, these processes exhibit a large scale uncertainty which can be softened by including the next-to-leading order (NLO) contributions, in which one-loop amplitudes have been a challenging component in processes of current importance $[1,2]$.

Recent years have seen rapid progress in computing one-loop amplitudes, due largely to the use of on-shell methods grouped under the name of "generalized unitarity." One advantage of on-shell methods is that they enable the computation of loop amplitudes in terms of tree-level amplitudes, which are relatively easier to generate either numerically or analytically in compact forms. 
Unitarity methods for one-loop amplitudes [3-18], recently reviewed in [19-21], depend on the knowledge of a small, canonical set of master integrals, in which any amplitude can be expanded linearly with coefficients that are rational functions of the kinematic invariants [22-37]. Generalized cuts of a loop integral are defined as the singularities in the limit of sets of virtual momenta going on shell: propagators are replaced by the delta functions of the on-shell conditions. For certain cuts, complex-valued momenta must be considered in order to solve all on-shell conditions. The coefficients of the master integrals are then obtained by matching generalized cuts of the loop amplitude and the master integrals.

Unitarity methods work most beautifully when all internal particles are massless. If massive particles are involved, the same cuts can be used to produce coefficients of most of the master integrals without any new conceptual difficulties. More significantly, there are some additional master integrals whose cuts are more difficult to solve. In the case of QCD processes involving $n$ massive quarks with masses $m_{i=1, \ldots, n}$, the additional master integrals are the tadpoles $A_{0}\left(m_{i}\right)$, and the "on-shell bubbles" $B_{0}\left(m_{i}^{2} ; m_{i}, 0\right)$ and $B_{0}\left(0 ; m_{i}, m_{i}\right)$. The massless counterparts of these integrals vanish in dimensional regularization. These bubbles are called on-shell because they are in the momentum channel of a single on-shell external particle, either the new massive particle or any of the massless ones. There are several proposals for computing some of these coefficients [12, 21, 38-42] either numerically or analytically, with or without unitarity cuts.

Here, in the context of renormalization, we will be concerned with the tadpole integral and the bubble $B_{0}\left(m^{2} ; m, 0\right)$, which has exactly the shape of the external leg correction diagram of the massive particle. Renormalized amplitudes also need the differentiated bubble, $B_{0}^{\prime}\left(m^{2} ; m, 0\right)$. The derivative is taken with respect to the first argument.

External leg correction diagrams contain an internal on-shell propagator, which implies the singularity of their cuts, and hence the singularity of the cuts of the amplitude. This problem was raised and nicely addressed in [18, 21], in the context of a numerical algorithm. The solution given in those papers was to omit the problematic contributions, taking care with the associated breaking of gauge invariance.

Here, we present a different solution. In the spirit of the unitarity method, we want to keep the ingredients of the cut as complete amplitudes, without discarding any contributions. We must therefore also include the corresponding counterterms. To regularize the singularity arising from the cut of the external leg correction diagram, we perform an off-shell continuation of the cut momentum. The divergence is cancelled between the cut loop diagram and the cut counterterm diagram, so we retain only the finite terms in the expansion around the on-shell kinematics. The examples given here involve 4-dimensional cuts, although the formalism is equally valid in $D$ dimensions. We are also able to sum over physical polarization states only, in the spinor-helicity formalism.

The paper is organized as follows. In section 2, we present the theoretical analysis. We define the off-shell continuation and show the cancellation of divergences for both bubble and tadpole coefficients from double and single cuts, respectively. In section 3, we illustrate the method with two examples computed with Feynman diagrams, namely $H \rightarrow b \bar{b}$ and $q \bar{q} \rightarrow t \bar{t}$. In section 4, we illustrate the method in the spinor-helicity formalism for one of the partial amplitudes in $\bar{t} t \rightarrow g g$. For this calculation, we make use of compact analytic 
formulas for tree amplitudes. In section 5, we summarize the algorithm. Appendix A lists the master integrals and some useful reduction formulas, and appendix B gives identities and formulas for the massive spinor formalism used in section 4.

\section{Description of the method}

The unitarity cut of the amplitude of a process with massive external particles should give information about the coefficients of the on-shell Green's function. Unfortunately the external leg correction diagrams are singular, because the propagator opposite the external leg carries the same momentum and is therefore also on shell.

In order to apply finite unitarity cuts, we perform an off-shell continuation by doing a double momentum shift. We shift the massive external momentum $k$ and one other external momentum $r$, to preserve momentum conservation. Assuming that both momenta are outgoing, the shift may be written as

$$
k \rightarrow \hat{k}=k+\xi \bar{k}, \quad r \rightarrow \hat{r}=r-\xi \bar{k} .
$$

We choose the momentum $\bar{k}$ such that $r \cdot \bar{k}=\bar{k}^{2}=0$ and $2(k \cdot \bar{k}) \neq 0$. The momentum $k$ is on shell: $k^{2}=m^{2}$, while $\hat{k}$ is off shell. The momentum $\hat{r}$ is still on shell after the shift. With this shift, the propagator opposite the external leg diverges as $1 / \xi$; thus the full amplitude diverges the same way. The cuts are calculated in terms of tree amplitudes. For the double cut, one has simply the three-point interaction $\mathcal{M}_{R}$, now continued off shell, and an $(n+1)$ point tree amplitude $\mathcal{M}_{L}$, on shell but depending on the parameter $\xi$. In the case of the single cut we have a single $(n+2)$-point off-shell tree amplitude $\mathcal{M}_{T}$, which is $\xi$-dependent. We expand $\mathcal{M}_{L}$ and $\mathcal{M}_{T}$ up to first order in $\xi$. The coefficients of this expansion could be obtained directly from a closed form for the full amplitude. Alternatively, separate recursive constructions could be used to generate them independently.

The external leg correction diagrams are exactly cancelled by the corresponding counterterms, provided that the renormalization constants are defined in the on-shell scheme. The counterterm diagrams are constructed from the renormalization constants and the various tree-level off-shell currents. The renormalization constants are known in terms of the master integrals. The other ingredients needed for this step are the various tree-level off-shell currents. An expansion in $\xi$ is performed here as well, and the divergent part is simply discarded since it is guaranteed to cancel the cut loop diagram. The off-shell currents are gauge-dependent. In the sum of all parts, gauge invariance is restored by construction: coefficients of master integrals are gauge invariant, and we have only added zero in the form of the external leg correction plus its counterterm.

After the cancellation of the divergent contributions, the on-shell limit is reached by setting $\xi=0$. Therefore, at every stage of the procedure, we systematically neglect terms of $\mathcal{O}(\xi)$.

In the remainder of this section, we demonstrate the cancellation of divergences in the cut between loop Feynman diagrams and the counterterm. Our conventions for the master integrals and their cuts are given in appendix A. 


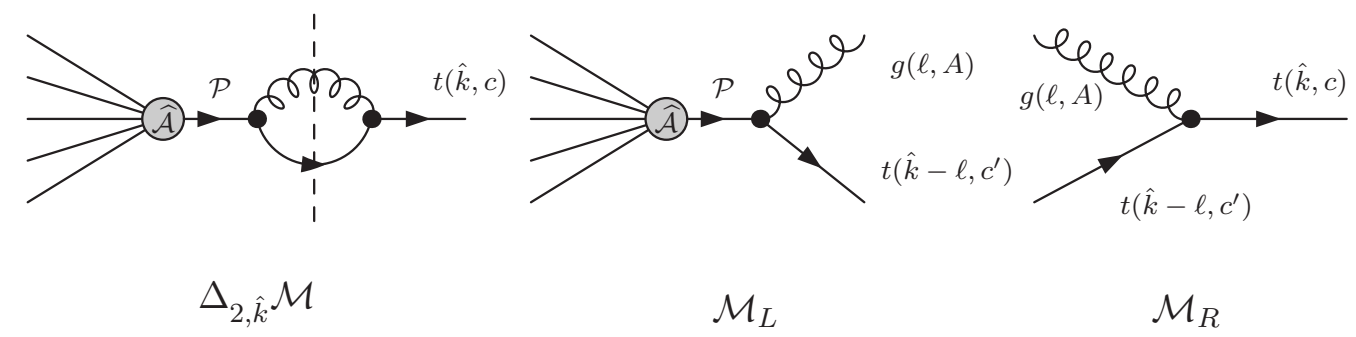

Figure 1. Double cut of the external leg correction diagram, and the left and right tree-level amplitudes. The cut momenta are $\ell$ and $k-\ell$. Color information is indicated by $c$ and $c^{\prime}$. The massive propagator giving the on-shell divergence is denoted by $\mathcal{P}$.

\subsection{Bubbles from double cut}

Consider the double cut of the external leg correction diagram for a massive fermion, as shown in figure 1 . Let $k$ denote the outgoing momentum of the external fermion, and $\ell$ and $k-\ell$ the momenta of the cut gluon and cut fermion, respectively.

The shifted massive propagator $\mathcal{P}$ is

$$
\mathcal{P}=\frac{i(m+\not k+\xi \bar{k})}{(k+\xi \bar{k})^{2}-m^{2}}=\frac{i(m+\not k+\xi \not k)}{\xi \gamma},
$$

where $\gamma$ is defined by

$$
\gamma \equiv 2 k \cdot \bar{k}
$$

The tree-level amplitudes $\mathcal{M}_{L}$ and $\mathcal{M}_{R}$ depicted in figure 1 read as follows:

$$
\begin{aligned}
\mathcal{M}_{L} & =\frac{g T_{c^{\prime} c^{\prime \prime}}^{A}}{(k+\xi \bar{k})^{2}-m^{2}}\left(\bar{u}_{k+\xi \bar{k}-\ell} \oint_{\ell}^{*}(m+\not k+\xi \bar{k}) \widehat{\mathcal{A}}_{c^{\prime \prime} c_{\mathrm{ext}}}\right), \\
\mathcal{M}_{R} & =-i g T_{c c^{\prime}}^{A}\left(\bar{u}_{k} \oint_{\ell} u_{k+\xi \bar{k}-\ell}\right) .
\end{aligned}
$$

Here $\widehat{\mathcal{A}}_{\mathcal{C}^{\prime \prime}} c_{\text {ext }}$ denotes the remaining parts of the diagram, including the external legs on the left and all color-flow information. ${ }^{1}$ Specifically, $c^{\prime \prime}$ denotes the color of the propagator $\mathcal{P}$.

Note that the external massive spinor $u_{k}$ is not being shifted. Its shift is possible but unnecessary, since terms arising from the $\mathcal{O}(\xi)$ contribution of $u_{k}$ drop out once the unrenormalized amplitude and the counterterm diagrams are summed together. We are performing the complete momentum shift on $\mathcal{M}_{L}$, supplemented by the specific instructions given above for how to continue the propagator $\mathcal{P}$ and the vertex $\mathcal{M}_{R}$.

Multiplying these expressions, we find that the double cut including the sum over polarization states, in Feynman gauge where $\sum \varepsilon_{\mu} \varepsilon_{\nu}^{*}=-g_{\mu \nu}$, is

$$
\frac{2 i g^{2} C_{F}}{\xi \gamma} \int d \mu_{2, \hat{k}}\left[\left(2 m^{2}-\xi \gamma\right)\left(\bar{u}_{k} \widehat{\mathcal{A}}_{c c_{\mathrm{ext}}}\right)+\left(\bar{u}_{k} \ell(m+\not k+\xi \not k) \widehat{\mathcal{A}}_{c c_{\mathrm{ext}}}\right)+m \xi\left(\bar{u}_{k} \not{k} \widehat{\mathcal{A}}_{c c_{\mathrm{ext}}}\right)\right] .
$$

\footnotetext{
${ }^{1}$ Since it depends on $\hat{k}$ and $\hat{r}, \widehat{\mathcal{A}}_{c^{\prime \prime}} c_{\text {ext }}$ depends on $\xi$.
} 
The double-cut integration measure $d \mu_{2, \hat{k}}$ is defined in equation (A.2), and the Dynkin index of the fundamental representation of $\mathrm{SU}(N)$ is

$$
C_{F}=\frac{N^{2}-1}{2 N}
$$

It accounts for the internal color sum, leaving a Kronecker delta function allowing us to replace $c^{\prime \prime}$ by $c$ in $\widehat{\mathcal{A}}_{c^{\prime \prime}} c_{\text {ext }}$.

Using integral reduction (see (A.4)), we get the bubble part of the diagram:

$$
\frac{g^{2} C_{F}}{16 \pi^{2} \xi \gamma}\left[4 m^{2}\left(\bar{u}_{k} \widehat{\mathcal{A}}_{c c_{\mathrm{ext}}}\right)+2 \xi m\left(\bar{u}_{k} \not \vec{k} \widehat{\mathcal{A}}_{c c_{\mathrm{ext}}}\right)\right] B_{0}\left(m^{2}+\xi \gamma ; m, 0\right) .
$$

Finally, we expand around $\xi=0$, using

$$
B_{0}\left(m^{2}+\xi \gamma ; m, 0\right)=B_{0}\left(m^{2} ; m, 0\right)+\xi \gamma B_{0}^{\prime}\left(m^{2} ; m, 0\right)
$$

and

$$
\widehat{\mathcal{A}}_{c c_{\mathrm{ext}}} \equiv \mathcal{A}_{c c_{\mathrm{ext}}}+\xi \mathcal{A}_{c c_{\mathrm{ext}}}^{\prime} .
$$

The result is that the bubble parts of the shifted diagram are given by

$$
\begin{aligned}
& \mathcal{M}_{B}=\frac{g^{2} C_{F}}{16 \pi^{2} \xi \gamma}\left\{4 m^{2} \xi \gamma\left(\bar{u}_{k} \mathcal{A}_{c c_{\text {ext }}}\right) B_{0}^{\prime}\left(m^{2} ; m, 0\right)\right.
\end{aligned}
$$

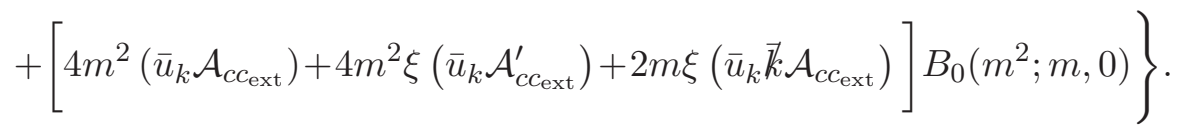

When the amplitudes are written in the spinor-helicity formalism [43-47], the procedure described above must be modified. Indeed, when using spinors, the completeness relation for polarization vectors is that of a light-like axial gauge rather than Feynman gauge:

$$
\sum_{\lambda= \pm} \varepsilon_{\mu} \varepsilon_{\nu}^{*}=-g_{\mu \nu}+\frac{\ell_{\mu} q_{\nu}+\ell_{\nu} q_{\mu}}{\ell \cdot q}
$$

Here $\ell$ is the momentum of the gluon, and $q$ is an arbitrary light-like "reference" momentum. The reference momentum (chosen independently for every gluon in the process) is needed to express polarization vectors in terms of spinors. Using eq. (2.8), we see that the double cut gets an extra $\mathcal{O}\left(\xi^{0}\right)$ contribution of the form

$$
\begin{aligned}
& -\frac{i g^{2} C_{F}}{\xi \gamma} \int d \mu_{2, \hat{k}}\left[\frac{\left(\bar{u}_{k} \ell u_{k+\xi \bar{k}-\ell}\right)\left(\bar{u}_{k+\xi \bar{k}-\ell} \not d(m+\not k+\xi \bar{k}) \widehat{\mathcal{A}}_{c c_{\mathrm{ext}}}\right)}{q \cdot \ell}\right. \\
& \left.+\frac{\left(\bar{u}_{k} \not u_{k+\xi \bar{k}-\ell}\right)\left(\bar{u}_{k+\xi \bar{k}-\ell} \ell(m+\not k+\xi \bar{k}) \widehat{\mathcal{A}}_{c c_{\mathrm{ext}}}\right)}{q \cdot \ell}\right] .
\end{aligned}
$$

The second of these terms vanishes in the sum over diagrams,

$$
\sum_{\widehat{\mathcal{A}}} \frac{1}{\xi \gamma}\left(\bar{u}_{k+\xi \bar{k}-\ell} \ell(m+\not k+\xi \vec{k}) \widehat{\mathcal{A}}_{c c_{\text {ext }}}\right),
$$




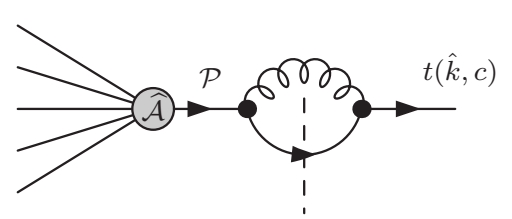

$\Delta_{1, \hat{k}} \mathcal{M}$

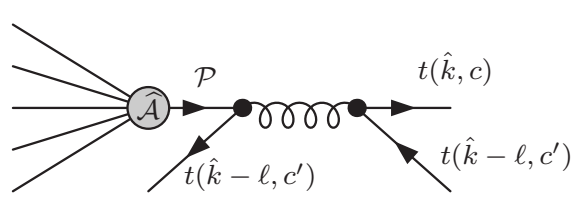

$\mathcal{M}_{T}$

Figure 2. Single cut of the external leg correction diagram.

due to the Ward identity with the on-shell cut gluon.

The remaining first term in (2.9) is equal to

$$
-\frac{i g^{2} C_{F}}{\gamma} \int d \mu_{2, \hat{k}} \frac{\bar{u}_{k} \vec{k}(m+\not k+\xi \vec{k}-\ell) \not l(m+\not k+\xi \vec{k}) \widehat{\mathcal{A}}_{c c_{\mathrm{ext}}}}{q \cdot \ell},
$$

which gives a contribution to the bubble part of the amplitude, (2.5), that is

$$
\frac{g^{2} C_{F}}{16 \pi^{2} \gamma} \frac{\bar{u}_{k} \not k \not k q(m+\not k) \mathcal{A}_{c c_{\mathrm{ext}}}}{q \cdot k} B_{0}\left(m^{2} ; m, 0\right) .
$$

This new contribution does not affect the divergent part of the bubble coefficient.

\subsection{Tadpole from single cut}

Now we consider the single cut of the massive propagator of the external leg correction diagram. See figure $2 .^{2}$ The ingredients are nearly the same as in the double cut, except that the polarization sum for the gluon is replaced by the propagator $-i g_{\mu \nu} / \ell^{2}$. The tree-level amplitude $\mathcal{M}_{T}$ is

$$
\mathcal{M}_{T}=-\frac{g^{2} C_{F}}{\ell^{2} \xi \gamma}\left(\bar{u}_{k} \gamma^{\mu} u_{k+\xi \bar{k}-\ell}\right)\left(\bar{u}_{k+\xi \bar{k}-\ell} \gamma_{\mu}(m+\not k+\xi \bar{k}) \widehat{\mathcal{A}}_{c^{\prime} c_{\mathrm{ext}}}\right) .
$$

The single cut $\Delta_{1, \hat{k}} \mathcal{M}$ reads as follows:

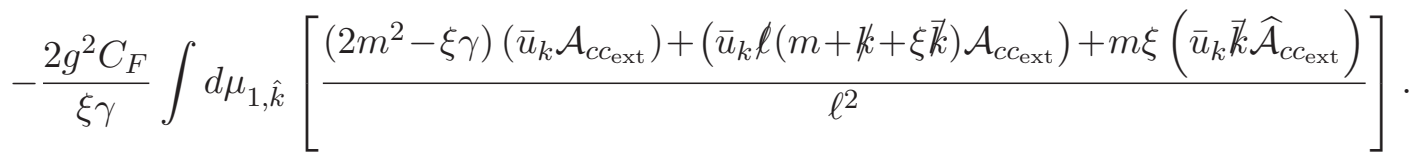

The single-cut integration measure $d \mu_{1, \hat{k}}$ is defined in equation (A.3). The integral reduction relations (A.5) allow us to compute the tadpole portion of the external leg correction diagram. The result is

$$
\frac{g^{2} C_{F}}{16 \pi^{2}}\left[\frac{\left(2 m^{2} \xi \gamma\right)\left(\bar{u}_{k} \widehat{\mathcal{A}}_{c c_{\mathrm{ext}}}\right)+\xi m\left(\bar{u}_{k} \vec{k} \widehat{\mathcal{A}}_{c c_{\mathrm{ext}}}\right)}{\xi \gamma\left(m^{2}+\xi \gamma\right)}\right] A_{0}(m) .
$$

\footnotetext{
${ }^{2}$ We do not compute the single cut of the gluon propagator since the massless tadpole vanishes in dimensional regularization.
} 


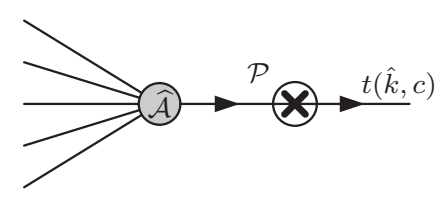

$\mathcal{M}^{\mathrm{ct}}$

Figure 3. Counterterm diagram.

Expanding in $\xi \rightarrow 0$, we get

$$
\mathcal{M}_{A}=\frac{g^{2} C_{F}}{16 \pi^{2}}\left[\left(\frac{2}{\xi \gamma}-\frac{1}{m^{2}}\right)\left(\bar{u}_{k} \mathcal{A}_{c c_{\mathrm{ext}}}\right)+\frac{1}{\gamma m}\left(\bar{u}_{k} \not \vec{k} \mathcal{A}_{c c_{\mathrm{ext}}}\right)+\frac{2}{\gamma}\left(\bar{u}_{k} \mathcal{A}_{c c_{\mathrm{ext}}}^{\prime}\right)\right] A_{0}(m)
$$

\subsection{Cancellation against the counterterm}

Now we write the external leg counterterm, $\mathcal{M}^{\text {ct }}$, depicted in figure 3 , in terms of the master integrals, and we show that the divergent part is exactly cancelled by the divergence of the external leg correction diagram.

The contribution of $\mathcal{M}^{\text {ct }}$ is

$$
\mathcal{M}^{\mathrm{ct}}=-\frac{1}{\xi \gamma}\left(\bar{u}_{k}\left(\not k \delta Z_{\psi}+\xi \bar{k} \delta Z_{\psi}-m \delta Z_{\psi}-m \delta Z_{m}\right)(\not k+\xi \not k+m) \widehat{\mathcal{A}}_{c c_{\mathrm{ext}}}\right) .
$$

In the on-shell scheme, the renormalization constants $\delta Z_{m}$ and $\delta Z_{\psi}$ are expressed in terms of master integrals as

$$
\begin{aligned}
& \delta Z_{m}=-\frac{g^{2} C_{F}}{16 \pi^{2}}\left[\frac{A_{0}(m)}{m^{2}}+2 B_{0}\left(m^{2} ; m, 0\right)\right] \\
& \delta Z_{\psi}=-\frac{g^{2} C_{F}}{16 \pi^{2}}\left[\frac{A_{0}(m)}{m^{2}}-4 m^{2} B_{0}^{\prime}\left(m^{2} ; m, 0\right)\right] .
\end{aligned}
$$

Therefore, the counterterm diagram is

$$
\mathcal{M}^{\text {ct }}=\mathcal{M}^{m}+\mathcal{M}^{\psi}
$$


where $\mathcal{M}^{m}$ is given by

$$
\begin{aligned}
\mathcal{M}^{m}=-\frac{g^{2} C_{F}}{16 \pi^{2}}\{ & {\left[\frac{2}{\xi \gamma} A_{0}(m)+\frac{4 m^{2}}{\xi \gamma} B_{0}\left(m^{2} ; m, 0\right)\right]\left(\bar{u}_{k} \widehat{\mathcal{A}}_{c c_{\mathrm{ext}}}\right) } \\
& \left.+\left[\frac{1}{\gamma m} A_{0}(m)+\frac{2 m}{\gamma} B_{0}\left(m^{2} ; m, 0\right)\right]\left(\bar{u}_{k} \vec{k} \widehat{\mathcal{A}}_{c c_{\mathrm{ext}}}\right)\right\} \\
\simeq-\frac{g^{2} C_{F}}{16 \pi^{2}}\{ & {\left[\frac{2}{\xi \gamma} A_{0}(m)+\frac{4 m^{2}}{\xi \gamma} B_{0}\left(m^{2} ; m, 0\right)\right]\left(\bar{u}_{k} \mathcal{A}_{c c_{\mathrm{ext}}}\right) } \\
& +\left[\frac{2}{\gamma} A_{0}(m)+\frac{4 m^{2}}{\gamma} B_{0}\left(m^{2} ; m, 0\right)\right]\left(\bar{u}_{k} \mathcal{A}_{c c_{\mathrm{ext}}}^{\prime}\right) \\
& \left.+\left[\frac{1}{\gamma m} A_{0}(m)+\frac{2 m}{\gamma} B_{0}\left(m^{2} ; m, 0\right)\right]\left(\bar{u}_{k} \vec{k} \mathcal{A}_{c c_{\mathrm{ext}}}\right)\right\},
\end{aligned}
$$

while $\mathcal{M}^{\psi}$ is given by

$$
\begin{aligned}
\mathcal{M}^{\psi} & =-\frac{g^{2} C_{F}}{16 \pi^{2}}\left\{\left[-\frac{1}{m^{2}} A_{0}(m)+4 m^{2} B_{0}^{\prime}\left(m^{2} ; m, 0\right)\right]\left(\bar{u}_{k} \widehat{\mathcal{A}}_{c c_{\mathrm{ext}}}\right)\right\} \\
& \simeq-\frac{g^{2} C_{F}}{16 \pi^{2}}\left\{\left[-\frac{1}{m^{2}} A_{0}(m)+4 m^{2} B_{0}^{\prime}\left(m^{2} ; m, 0\right)\right]\left(\bar{u}_{k} \mathcal{A}_{c c_{\mathrm{ext}}}\right)\right\} .
\end{aligned}
$$

When the spinor-helicity formalism is used, the extra contribution (2.12) is accounted for by adding the following term to eq. (2.16):

$$
\mathcal{M}^{k}=-\frac{1}{\xi \gamma} \bar{u}_{k}\left[(\not k+\xi k-m)(\not k+\xi \not k) q \delta Z_{k}^{\prime}\right](\not k+\xi \not k+m) \widehat{\mathcal{A}}_{c c_{\mathrm{ext}}},
$$

where

$$
\delta Z_{k}^{\prime}=\frac{g^{2} C_{F}}{16 \pi^{2}} \frac{B_{0}\left(m^{2} ; m, 0\right)}{q \cdot k} .
$$

Having expanded the counterterm around $\xi=0$ at $\mathcal{O}\left(\xi^{0}\right)$, it is straightforward to check the cancellation of the terms proportional to $\xi^{-1}$ and the ones which depend on $\bar{k}$ once $\mathcal{M}_{A}, \mathcal{M}_{B}$, and $\mathcal{M}^{\text {ct }}$ are added together. The sum is identically zero, since in the on-shell scheme the external leg correction diagram is exactly compensated by the external leg counterterm. The actual contribution to the tadpole and bubble coefficients comes from the other diagrams in the full amplitude. They are finite in $\xi$, thus no $\bar{k}$ dependence arises in the $\xi \rightarrow 0$ limit.

\section{$3 \quad$ Examples}

\subsection{The process $H \rightarrow b \bar{b}$}

As a first application, we focus on a simple process, the Higgs decay into a bottom-antibottom pair:

$$
H\left(k_{1}\right) \rightarrow b\left(k_{2}, c_{2}\right) \bar{b}\left(k_{3}, c_{3}\right)
$$



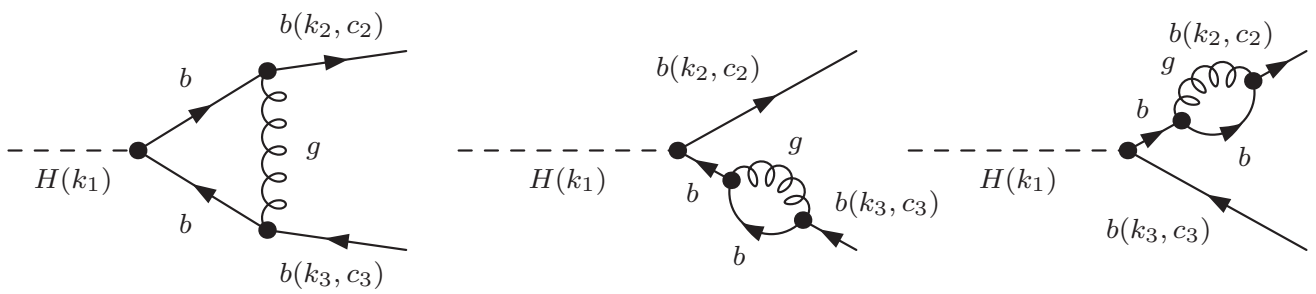

Figure 4. Virtual corrections to the process (3.1).

We apply the procedure described in section 2 to compute the coefficients of the on-shell bubble $B_{0}\left(m_{b}^{2} ; m_{b}, 0\right)$, of $B_{0}^{\prime}\left(m_{b}^{2} ; m_{b}, 0\right)$ and of the tadpole $A_{0}\left(m_{b}\right)$ entering the NLO QCD amplitude $\mathcal{M}_{H \rightarrow b \bar{b}}$ [48-51]. We regularize the tree-level amplitudes using the following shift:

$$
k_{2} \rightarrow \hat{k}_{2}=k_{2}+\xi \bar{k}, \quad k_{3} \rightarrow \hat{k}_{3}=k_{3}-\xi \bar{k} .
$$

The massless momentum $\bar{k}$ is such that

$$
\gamma_{2} \equiv 2\left(k_{2} \cdot \bar{k}\right) \neq 0, \quad \gamma_{3} \equiv 2\left(k_{3} \cdot \bar{k}\right) \neq 0,
$$

while $k_{2}$ and $k_{3}$ are on-shell: $k_{2}^{2}=k_{3}^{2}=m_{b}^{2}$. Here we use a slightly different shift since both the shifted momenta are off shell. In this example, the modification of the method is irrelevant and streamlines the computation.

The coefficient $b_{B_{0}\left(m_{b}^{2}+\gamma_{2} \xi ; m_{b}, 0\right)}$ of $B_{0}\left(m_{b}^{2}+\gamma_{2} \xi ; m_{b}, 0\right)$ can be computed by means of the double cut,

$$
\Delta_{2, \hat{k}_{2}} \mathcal{M}_{H \rightarrow b \bar{b}}=\int d \mu_{2, \hat{k}_{2}} \mathcal{M}_{H \rightarrow b g \bar{b}}\left(k_{1}, \hat{k}_{2}-\ell, \ell, \hat{k}_{3}\right) \mathcal{M}_{g b \rightarrow b}\left(\ell, \hat{k}_{2}-\ell, \hat{k}_{2}\right),
$$

where the tree-level amplitudes on the r.h.s. are related to the processes

$$
H\left(k_{1}\right) \rightarrow b\left(k_{2}, c_{2}\right) g\left(k_{4}, A_{4}\right) \bar{b}\left(k_{3}, c_{3}\right), \quad g\left(k_{1}, A_{1}\right) b\left(k_{2}, c_{2}\right) \rightarrow b\left(k_{3}, c_{3}\right),
$$

respectively. Up to $\mathcal{O}(\xi)$, the relevant part of the double cut is given by

$$
\begin{aligned}
& -\frac{8 g^{2} e m_{b} \delta_{c_{3}}^{c_{2}}}{3 M_{W} s_{\mathrm{w}}} \int d \mu_{2, k_{2}}\left\{\frac{m\left(\bar{u}_{k_{2}} \ell v_{k_{3}}\right)+\left(k_{1} \cdot \ell\right)\left(\bar{u}_{k_{2}} v_{k_{3}}\right)}{\left(k_{3}+\ell\right)^{2}-m_{b}^{2}}\right. \\
& \left.+\frac{\left(\bar{u}_{k_{2}} \ell(k / 2+\xi \bar{k}) v_{k_{3}}\right)+m\left(\bar{u}_{k_{2}}\left(\ell+k_{2}+\xi \vec{k}\right) v_{k_{3}}\right)-\left(\gamma_{2} \xi-m_{b}^{2}\right)\left(\bar{u}_{k_{2}} v_{k_{3}}\right)}{2 \gamma_{2} \xi}+\cdots\right\} .
\end{aligned}
$$

Using the results (A.4), we get the coefficient

$$
\begin{aligned}
& b_{B_{0}\left(m_{b}^{2}+\gamma_{2} \xi ; m_{b}, 0\right)}=\frac{g^{2} e m_{b} \delta_{c_{3}}^{c_{2}} i}{24 \pi^{2} M_{W} s_{\mathrm{w}}}\left\{\left[\frac{2\left(2 m_{b}^{2}-M_{H}^{2}\right)}{4 m_{b}^{2}-M_{H}^{2}}-\frac{\gamma_{2} \xi-2 m_{b}^{2}}{\gamma_{2} \xi}\right]\left(\bar{u}_{k_{2}} v_{k_{3}}\right)\right. \\
& \left.\frac{m_{b}\left(2 m_{b}^{2}+3 \gamma_{2} \xi\right)}{\left(m_{b}^{2}+\gamma_{2} \xi\right) \gamma_{2} \xi}\left[m_{b}\left(\bar{u}_{k_{2}} v_{k_{3}}\right)+\xi\left(\bar{u}_{k_{2}} \not k v_{k_{3}}\right)\right]\right\} \text {. }
\end{aligned}
$$


The parameter $e$ is the charge of the positron, while $M_{W}$ and $s_{\mathrm{w}}$ are the mass of the $W$-boson and the sine of the weak mixing angle respectively.

The coefficient $b_{B_{0}\left(m_{b}^{2}-\xi \gamma_{3} ; m_{b}, 0\right)}$ of $B_{0}\left(m_{b}^{2}-\xi \gamma_{3} ; m_{b}, 0\right)$ can be obtained analogously starting from the double cut

$$
\Delta_{2, \hat{k}_{3}} \mathcal{M}_{H \rightarrow b \bar{b}}=\int d \mu_{2, \hat{k}_{3}} \mathcal{M}_{H \rightarrow b g \bar{b}}\left(k_{1}, \hat{k}_{2}, \ell, \hat{k}_{3}-\ell\right) \mathcal{M}_{g \bar{b} \rightarrow \bar{b}}\left(\ell, \hat{k}_{3}-\ell, \hat{k}_{3}\right)
$$

and using the relations (A.4). $\mathcal{M}_{g \bar{b} \rightarrow \bar{b}}$ is the tree-level amplitude of the process

$$
g\left(k_{1}, A_{1}\right) \bar{b}\left(k_{2}, c_{2}\right) \rightarrow \bar{b}\left(k_{3}, c_{3}\right)
$$

The result is

$$
\begin{aligned}
b_{B_{0}\left(m_{b}^{2}-\gamma_{3} \xi ; m_{b}, 0\right)}=\frac{g^{2} e m_{b} \delta_{c_{3}}^{c_{2}} i}{24 \pi^{2} M_{W} s_{\mathrm{w}}}\{ & {\left[\frac{2\left(2 m_{b}^{2}-M_{H}^{2}\right)}{4 m_{b}^{2}-M_{H}^{2}}-\frac{2 m_{b}^{2}+\gamma_{3} \xi}{\gamma_{3} \xi}\right]\left(\bar{u}_{k_{2}} v_{k_{3}}\right) } \\
& \left.+\frac{m_{b}\left(3 \gamma_{3} \xi-2 m_{b}^{2}\right)}{\gamma_{3} \xi\left(m_{b}^{2}-\gamma_{3} \xi\right)}\left[\xi\left(\bar{u}_{k_{2}} \vec{k} v_{k_{3}}\right)+m_{b}\left(\bar{u}_{k_{2}} v_{k_{3}}\right)\right]\right\}
\end{aligned}
$$

The coefficients of $B_{0}\left(m_{b}^{2} ; m_{b}, 0\right)$ and of $B_{0}^{\prime}\left(m_{b}^{2} ; m_{b}, 0\right)$ can be obtained by expanding

$$
b_{B_{0}\left(m_{b}^{2}+\gamma_{2} \xi ; m_{b}, 0\right)} B_{0}\left(m_{b}^{2}+\gamma_{2} \xi ; m_{b}, 0\right)+b_{B_{0}\left(m_{b}^{2}-\gamma_{3} \xi ; m_{b}, 0\right)} B_{0}\left(m_{b}^{2}-\gamma_{3} \xi ; m_{b}, 0\right)
$$

around $\xi=0$ and neglecting terms of $\mathcal{O}(\xi)$.

The tadpole coefficient $a_{A_{0}\left(m_{b}\right)}$ can be computed from the sum of two single cut diagrams,

$$
\Delta_{1, \hat{k}_{2}} \mathcal{M}_{H \rightarrow b \bar{b}}+\Delta_{1,-\hat{k}_{3}} \mathcal{M}_{H \rightarrow b \bar{b}}=\int d \mu_{1,0} \mathcal{M}_{H b \rightarrow b b \bar{b}}\left(k_{1}, \ell, \hat{k}_{2}, \ell, \hat{k}_{3}\right)
$$

provided that the loop momentum $\ell$ is chosen appropriately. ${ }^{3}$ The tree-level amplitude on the r.h.s. is related to the process

$$
H\left(k_{1}\right) b\left(k_{2}, c\right) \rightarrow b\left(k_{3}, c_{3}\right) b\left(k_{4}, c\right) \bar{b}\left(k_{5}, c_{5}\right) .
$$

Divergent diagrams with zero-momentum internal gluons do not contribute, owing to the color flow of the process (3.11). At $\mathcal{O}(\xi)$, the part of the single cut (3.10) which is relevant for the computation of the tadpole coefficient reads as follows:

$$
\begin{aligned}
-\frac{4 g^{2} e m_{b} \delta_{c_{3}}^{c_{2}} i}{3 M_{W} s_{\mathrm{w}}} \int d \mu_{1,0}\{ & \frac{\left(\bar{u}_{k_{2}} \ell\left(k_{2}+\xi \bar{k}\right) v_{k_{3}}\right)+m_{b}\left(\bar{u}_{k_{2}} \ell v_{k_{3}}\right)}{\left(\ell+k_{2}+\xi \bar{k}\right)^{2} \gamma_{2} \xi} \\
& \left.-\frac{m_{b}\left(\bar{u}_{k_{2}} \ell v_{k_{3}}\right)-\left(\bar{u}_{k_{2}}\left(k_{3}-\xi \bar{k}\right) \ell v_{k_{3}}\right)}{\left(\ell+k_{3}-\xi \bar{k}\right)^{2} \gamma_{3} \xi}+\cdots\right\} .
\end{aligned}
$$

\footnotetext{
${ }^{3}$ In particular in each diagram of figure 4 the loop momentum has to be fixed such that the only internal propagator appearing are $\ell^{2},\left(\ell+\hat{k}_{2}\right)^{2}-m_{b}^{2}$, and $\left(\ell-\hat{k}_{3}\right)^{2}-m_{b}^{2}$.
} 

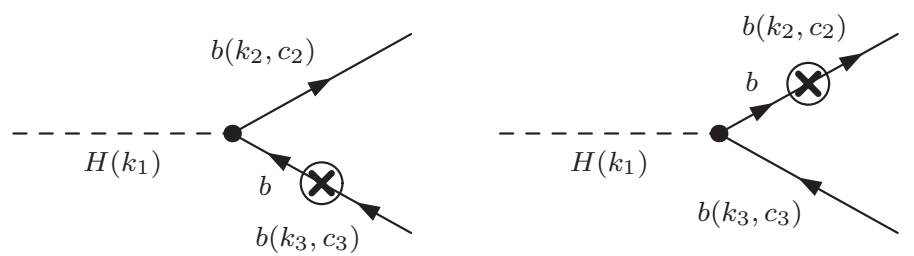

Figure 5. External leg counterterm diagrams of the process (3.1).

The coefficient $a_{A_{0}\left(m_{b}\right)}$ is obtained using the relations (A.5) and expanding the following expression in $\xi$ :

$$
\begin{aligned}
\frac{g^{2} e m_{b} \delta_{c}^{c_{2}} i}{24 \pi^{2} M_{W} s_{\mathrm{w}}}\{ & {\left[m_{b} \xi\left(\bar{u}_{k_{2}} \vec{k} v_{k_{3}}\right)+m_{b}^{2}\left(\bar{u}_{k_{2}} v_{k_{3}}\right)\right]\left[\frac{1}{\left(m_{b}^{2}+\gamma_{2} \xi\right) \gamma_{2} \xi}\right.} \\
& \left.\left.-\frac{1}{\left(m_{b}^{2}-\gamma_{3} \xi\right) \gamma_{3} \xi}\right]+\left(\frac{1}{\gamma_{2} \xi}-\frac{1}{\gamma_{3} \xi}\right)\left(\bar{u}_{k_{2}} v_{k_{3}}\right)\right\} .
\end{aligned}
$$

The results (3.6), (3.8), and (3.13), have been checked performing Passarino-Veltman decomposition of the one loop amplitude obtained from the Feynman diagrams in figure 4. This procedure has been performed with the help of FeynArts [52], FormCalc [53, 54] and FeynCalc [55].

We have verified the cancellation of the $\mathcal{O}\left(\xi^{-1}\right)$ terms against the contribution of the two counterterm diagrams depicted in figure 5, along the lines of section 2.3. In the $\xi \rightarrow 0$ limit, the terms containing $\bar{k}$ drop out. The bubble and the tadpole coefficients are thus independent of this unphysical momentum.

\subsection{The process $q \bar{q} \rightarrow t \bar{t}$}

A less trivial example involves the process of top-anti-top production via quark-anti-quark annihilation:

$$
q\left(k_{1}, c 1\right) \bar{q}\left(k_{2}, c_{2}\right) \rightarrow t\left(k_{3}, c_{3}\right) \bar{t}\left(k_{4}, c_{4}\right), \quad(q \neq t) .
$$

This is one among the channels entering the hadronic production of a top-anti-top pair whose NLO QCD contributions have been computed [56-59] and implemented in MCFM [60] and in MC@NLO [61]. We have computed the coefficient of $B_{0}\left(m_{t}^{2} ; m_{t}, 0\right), B_{0}^{\prime}\left(m_{t}^{2} ; m_{t}, 0\right)$ and of the tadpole $A_{0}\left(m_{t}\right)$. The tree-level amplitudes have been regularized using the shift

$$
k_{3} \rightarrow \hat{k}_{3}=k_{3}+\xi \bar{k}, \quad k_{4} \rightarrow \hat{k}_{4}=k_{4}-\xi \bar{k} .
$$

We have used the double cuts

$$
\begin{aligned}
& \Delta_{2, \hat{k}_{3}} \mathcal{M}_{q \bar{q} \rightarrow t \bar{t}}=\int d \mu_{2, \hat{k}_{3}} \mathcal{M}_{q \bar{q} \rightarrow t g \bar{t}}\left(k_{1}, k_{2}, \hat{k}_{3}-\ell, \ell, \hat{k}_{4}\right) \mathcal{M}_{g t \rightarrow t}\left(\ell, \hat{k}_{3}-\ell, \hat{k}_{3}\right), \\
& \Delta_{2, \hat{k}_{4}} \mathcal{M}_{q \bar{q} \rightarrow t \bar{t}}=\int d \mu_{2, \hat{k}_{4}} \mathcal{M}_{q \bar{q} \rightarrow t g \bar{t}}\left(k_{1}, k_{2}, \hat{k}_{3}, \ell, \hat{k}_{4}-\ell\right) \mathcal{M}_{g \bar{t} \rightarrow \bar{t}}\left(\ell, \hat{k}_{4}-\ell, \hat{k}_{4}\right),
\end{aligned}
$$



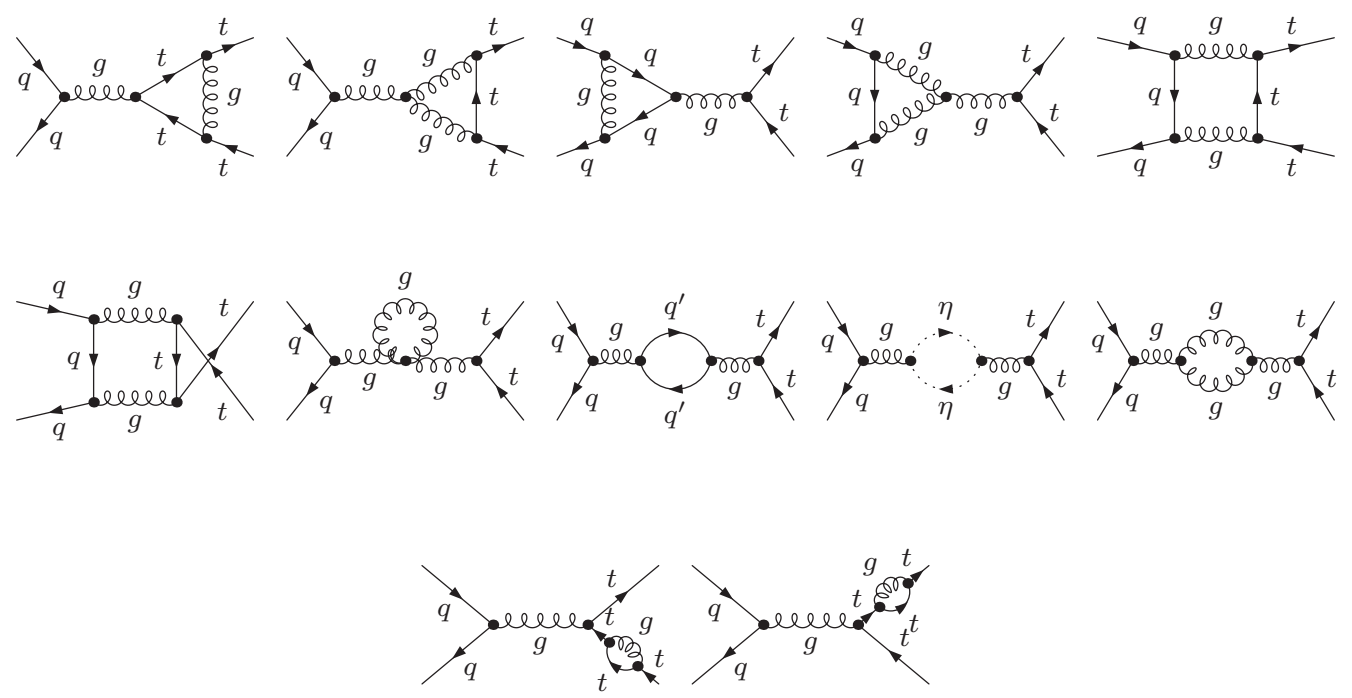

Figure 6. NLO QCD corrections to the process (3.14). The ghosts are labelled by $\eta$ while $q^{\prime}=$ $u, d, c, s, t, b$.

the single cut

$$
\Delta_{1, \hat{k}_{3}} \mathcal{M}_{q \bar{q} \rightarrow t \bar{t}}+\Delta_{1,-\hat{k}_{4}} \mathcal{M}_{q \bar{q} \rightarrow t \bar{t}}=\int d \mu_{1,0} \mathcal{M}_{q \bar{q} t \rightarrow t t \bar{t}}\left(k_{1}, k_{2}, \ell, \hat{k}_{3}, \ell, \hat{k}_{4}\right),
$$

and the relations (A.4) and (A.5). The coefficients can be obtained performing an expansion around $\xi=0$ at $\mathcal{O}\left(\xi^{0}\right)$, along the lines of section 3.1. The actual expression of the coefficients is lengthy and is omitted. We have checked our result against the Passarino-Veltman based computation of the Feynman diagrams depicted in figure 6. In the renormalized amplitude, we have checked the cancellation of the $\mathcal{O}\left(\xi^{-1}\right)$ contributions and of the terms containing the massless momentum $\bar{k}$.

\section{On-shell bubbles in the spinor-helicity formalism}

In this section we illustrate our method in the spinor-helicity formalism. We compute the bubble coefficient of an amplitude from the usual cut integral using double cut spinor integration [62-67].

As discussed in section 2, the spinor-helicity formalism requires a gauge choice of a null "reference" momentum for each gluon. On-shell quantities are independent of this choice, but it plays a role in our method because of the off-shell continuation. Of course, the total bubble coefficient will be gauge-invariant. However, the independence of gauge choice will not be apparent until all counterterm diagrams are considered, including the internal ones.

The cut integral involves the off-shell continuation of a three-point amplitude which is a varying function of $q$, the reference momentum for the cut gluon. Furthermore, the off-shell current $\widehat{\mathcal{A}}_{c c_{\text {ext }}}$ (defined in section 2.1) depends on the reference momenta chosen for the external gluons. 
In practice, one makes a convenient choice of reference momenta. It is important to be consistent in this choice throughout the entire calculation. The gauge choice of the reference momentum $q$ of the cut gluon cancels out immediately between the cut and its counterterm. In the following example, we keep $q$ general to show this cancellation, while making convenient choices for the reference momenta of external gluons.

The spinor-helicity formalism can accommodate massive particles as follows [45, 68]. For a particle of mass $m$ and momentum $p$, the massive spinors are given by

$$
\begin{array}{ll}
|p\rangle=\frac{(\not p+m) \mid \eta]}{\left[p^{b} \eta\right]}, & \mid p]=\frac{(\not p+m)|\eta\rangle}{\left\langle p^{b} \eta\right\rangle}, \\
\langle p|=\frac{[\eta \mid(\not p+m)}{\left[\eta p^{b}\right]}, & {\left[p \mid=\frac{\langle\eta|(\not p+m)}{\left\langle\eta p^{b}\right\rangle},\right.}
\end{array}
$$

where $\eta$ is a fixed null momentum, and we define the null vector

$$
p^{b}=p-\frac{m^{2}}{2 p \cdot \eta} \eta
$$

For an antiparticle, the mass $m$ should be replaced by $-m$ everywhere.

It follows that massive spinor products obey the following (anti)symmetry relations:

$$
\langle i j\rangle=-\langle j i\rangle, \quad[i j]=-[j i], \quad\langle i j]=[j i\rangle .
$$

See appendix B for further details.

As an example, we now focus on the process

$$
\bar{t}\left(k_{1}, c_{1}, h_{1}\right) t\left(k_{2}, c_{2}, h_{3}\right) \rightarrow g\left(k_{3}, c_{3}, h_{3}\right) g\left(k_{4}, c_{4}, h_{4}\right),
$$

where $h_{i}\left(c_{i}\right)$ is the helicity (color) of the $i^{t h}$ particle. We will compute the coefficient of $B_{0}\left(m_{t}^{2} ; m_{t}, 0\right)$ of the leading-color part of the all-minus helicity amplitude. Contributions to the on-shell bubble coefficients come from the unrenormalized amplitude $\mathcal{M}_{\bar{t} t \rightarrow g g}^{(1)}$ in the $k_{1}^{2}$ and $k_{2}^{2}$ channels and from the counterterm diagrams.

\subsection{Contributions of the unrenormalized amplitude}

At one loop and in $D=4$ dimensions, the color decomposition of the unrenormalized amplitude reads as follows $[69,70]$ :

$$
\begin{gathered}
\mathcal{M}_{\bar{t} t \rightarrow g g}^{(1)}=\frac{2 g^{4}}{(4 \pi)^{2}}\left[\sum_{(i, j) \in\{(3,4),(4,3)\}} N\left(T^{c_{i}} T^{c_{j}}\right)_{c_{2}}^{c_{1}} A_{4 ; 1}^{(1)}\left(1_{\bar{t}}^{h_{1}}, 2_{t}^{h_{2}}, i_{g}^{h_{i}}, j_{g}^{h_{j}}\right)\right. \\
\left.+\operatorname{tr}\left(T^{c_{3}} T^{c_{4}}\right) \delta_{c_{2}}^{c_{1}} A_{4 ; 3}^{(1)}\left(1_{\bar{t}}^{h_{1}}, 2_{t}^{h_{2}}, 3_{g}^{h_{3}}, 4_{g}^{h_{4}}\right)\right],
\end{gathered}
$$

where $N$ is the number of colors. The one-loop partial amplitudes $A_{4 ; 1}^{(1)}$ are the leading-color ones, while $A_{4 ; 3}^{(1)}$ is subleading. ${ }^{4}$

\footnotetext{
${ }^{4}$ The factor of 2 is related to the different normalization of the color matrices used through the paper and the ones in the color-ordered Feynman rules as in [71].
} 


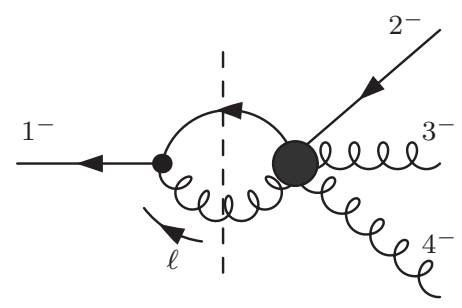

$\Delta_{2, k} A^{R}$

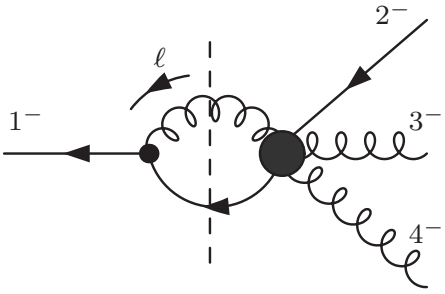

$\Delta_{2, k} A^{L}$

Figure 7. $k_{1}^{2}$-channel cut of the right and left primitive amplitudes.

The external leg corrections of the anti-top are leading color, thus we focus on the leading-color partial amplitudes. We compute the bubble coefficient of $A_{4 ; 1}^{(1)}\left(1_{\bar{t}}^{-}, 2_{t}^{-}, 3_{g}^{-}, 4_{g}^{-}\right)$. The coefficient of $A_{4 ; 1}^{(1)}\left(1_{\bar{t}}^{-}, 2_{t}^{-}, 4_{g}^{-}, 3_{g}^{-}\right)$can be obtained from the previous one via the substitution $3 \leftrightarrow 4$. The leading-color partial amplitude can be written in terms of primitive amplitudes as [69]

$$
\begin{aligned}
A_{4 ; 1}^{(1)}\left(1_{\bar{t}}^{-}, 2_{t}^{-}, 3_{g}^{-}, 4_{g}^{-}\right)= & A^{L}\left(1_{\bar{t}}^{-}, 2_{t}^{-}, 3_{g}^{-}, 4_{g}^{-}\right)-\frac{1}{N^{2}} A^{R}\left(1_{\bar{t}}^{-}, 2_{t}^{-}, 3_{g}^{-}, 4_{g}^{-}\right) \\
& +\frac{1}{N} \sum_{q} A_{q}^{L,[1 / 2]}\left(1_{\bar{t}}^{-}, 2_{t}^{-}, 3_{g}^{-}, 4_{g}^{-}\right),
\end{aligned}
$$

where the sum runs over the quark species. The primitive amplitudes contributing to the coefficient we are interested in are the left amplitude $A^{L}$ and the right amplitude $A^{R}$. They can be obtained starting from the color-ordered Feynman rules [71] and selecting the diagrams without a closed fermion loop, where the fermion flow passes to the left $\left(A^{L}\right)$ or the right $\left(A^{R}\right)$ of the loop.

\subsubsection{The cut in the $k_{1}^{2}$ channel}

We perform the shift setting $\bar{k}=k_{4}$, i.e.

$$
k_{1} \rightarrow \hat{k}_{1}=k_{1}+\xi k_{4}, \quad k_{4} \rightarrow \hat{k}_{4}=(1+\xi) k_{4}, \quad \gamma=\langle 4|1| 4] .
$$

Note that these shifts have the same sign, since $k_{1}$ is incoming while $k_{4}$ is outgoing, in contrast to our all-outgoing convention of section 2 .

The double cut of the right amplitude $A^{R}$, figure 7 , reads as follows:

$$
\Delta_{2,-\hat{k}_{1}} A^{R}\left(1_{\bar{t}}^{-}, 2_{t}^{-}, 3_{g}^{-}, 4_{g}^{-}\right)=\int d \mu_{2,-\hat{k}_{1}} I^{R} .
$$

The integrand $I^{R}$ is written in terms of color-ordered tree-level helicity amplitudes as

$$
I^{R}=\sum_{h_{t}, h_{g}= \pm} A\left(1_{\bar{t}},-\left(\ell+\hat{k}_{1}\right)_{t}^{-h_{t}},-\ell_{g}^{-h_{g}}\right) A\left(\left(\ell+\hat{k}_{1}\right)_{\bar{t}}^{h_{t}}, 2_{t}^{-}, 3_{g}^{-}, 4_{g}^{-}, \ell_{g}^{h_{g}}\right) .
$$


The necessary helicity amplitudes are collected in appendix B.2.

After the spinor integration and the $\xi$ expansion, the bubble coefficient $b_{B_{0}\left(m_{t}^{2}+\xi \gamma ; m_{t}, 0\right)}^{R}$ can be written in a relatively compact form:

$$
\begin{aligned}
& b_{B_{0}\left(m_{t}^{2}+\xi \gamma ; m_{t}, 0\right)}^{R}= \frac{1}{\xi} \frac{2 m_{t}^{3}\langle 34\rangle[12]}{[34]\langle 4|1| 4]^{2}}+\frac{m_{t}^{2}\langle 34\rangle\left(m_{t}[2 q]\langle q 4\rangle[41]+[14\rangle\langle q|1| 2][4 q]\right)}{[34]\langle 4|1| 4]^{2}\langle q|1| q]} \\
&-\frac{m_{t}}{[34]\langle 4|1| 4]^{2}}\left[m_{t}\langle 41][42]\langle 34\rangle+m_{t}^{2}\langle 32]\langle 41]+m_{t}\langle 4|1| 2][13\rangle\right. \\
&-2[12]\langle 34\rangle\langle 4|1| 4]] .
\end{aligned}
$$

The dependence on $q$ comes from the off-shell three-point function. See equations (B.9) and (B.10). This dependence will drop out in the sum with the counterterm diagram.

The double cut of the left amplitude $A^{L}$ reads as follows (cf. figure 7):

$$
\Delta_{2,-\hat{k}_{1}} A^{L}\left(1_{\bar{t}}^{-}, 2_{t}^{-}, 3_{g}^{-}, 4_{g}^{-}\right)=\int d \mu_{2,-\hat{k}_{1}} I^{L}
$$

with the integrand $I^{L}$ defined as

$$
I^{L}=\sum_{h_{t}, h_{g}= \pm} A\left(1_{\bar{t}},-\ell_{g}^{-h_{g}},-\left(\ell+\hat{k}_{1}\right)_{t}^{-h_{t}}\right) A\left(\left(\ell+\hat{k}_{1}\right)_{\bar{t}}^{h_{t}}, \ell_{g}^{h_{g}}, 2_{t}^{-}, 3_{g}^{-}, 4_{g}^{-}\right) .
$$

By considering the properties of the color-ordered Feynman rules, the $\bar{q} g q g g$ amplitude can be expressed as a linear combination of $\bar{q} q g g g$ amplitudes, as depicted in figure 8 . Therefore $I^{L}$ can be written as $I^{L}=I^{R}+I^{l}$, where $I^{l}$ is given by

$$
\begin{aligned}
I^{l}=\sum_{h_{t}, h_{g}= \pm} A\left(1_{\bar{t}},-\left(\ell+\hat{k}_{1}\right)_{t}^{-h_{t}},-\ell_{g}^{-h_{g}}\right)\left[A\left(\left(\ell+\hat{k}_{1}\right)_{\bar{t}}^{h_{t}}, 2_{t}^{-}, 3_{g}^{-}, \ell_{g}^{h_{g}}, 4_{g}^{-}\right)\right. \\
\left.+A\left(\left(\ell+\hat{k}_{1}\right)_{\bar{t}}^{h_{t}}, 2_{t}^{-}, \ell_{g}^{h_{g}}, 3_{g}^{-}, 4_{g}^{-}\right)\right] .
\end{aligned}
$$

It turns out that the bubble coefficient from $I^{l}$ vanishes. Therefore, the bubble coefficient of the left amplitude, $b_{B_{0}\left(m_{t}^{2}+\xi \gamma ; m_{t}, 0\right)}^{L}$ is equal to the one of the right amplitude:

$$
b_{B_{0}\left(m_{t}^{2}+\xi \gamma ; m_{t}, 0\right)}^{L}=b_{B_{0}\left(m_{t}^{2}+\xi \gamma ; m_{t}, 0\right)}^{R} .
$$

The final contribution to the bubble coefficient of the leading color part of the amplitude (4.6) is obtained using the results (4.11) and (4.15) and expanding $B_{0}\left(m_{t}^{2}+\xi \gamma ; m_{t}, 0\right)$ around $\xi=0$. The outcome is

$$
\begin{aligned}
2 g^{2}\left\{\frac{g^{2} C_{F}}{8 \pi^{2}}\left(T^{c_{3}} T^{c_{4}}\right)_{c_{2}}^{c_{1}}\right. & {\left[b_{B_{0}\left(m_{t}^{2}+\xi \gamma ; m_{t}, 0\right)}^{R} B_{0}\left(m_{t}^{2} ; m_{t}, 0\right)\right.} \\
& \left.\left.-\frac{2 m_{t}^{3}\langle 34\rangle[12]}{[34]\langle 4|1| 4]} B_{0}^{\prime}\left(m_{t}^{2} ; m_{t}, 0\right)\right]+(3 \leftrightarrow 4)\right\} .
\end{aligned}
$$




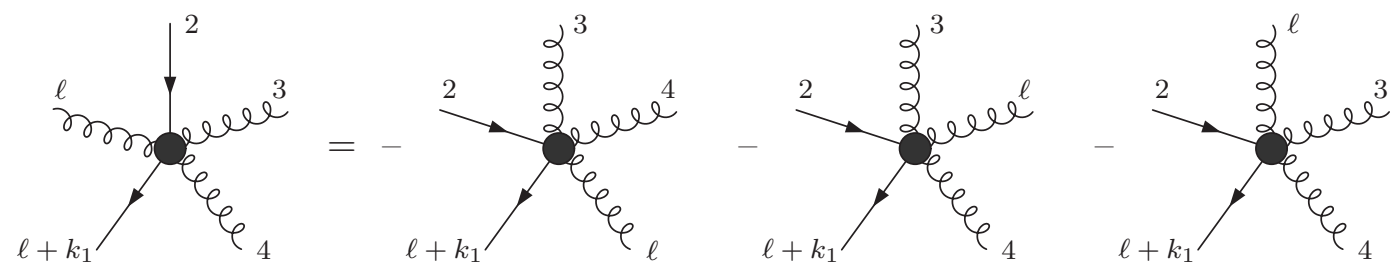

Figure 8. Pictorial representation of the relation between the $\bar{q} g q g g$ and the $\bar{q} q g g g$ color-ordered tree-level amplitudes.

\subsubsection{The cut in the $k_{2}^{2}$ channel}

In this case, the symmetry of the amplitude allows us to write down the result as a simple relabeling of the contribution from the $k_{1}^{2}$ channel. It suffices to exchange $1 \leftrightarrow 2,3 \leftrightarrow 4$, and $m_{t} \rightarrow-m_{t}$. The result is

$$
\begin{aligned}
2 g^{2}\left\{\frac{g^{2} C_{F}}{8 \pi^{2}}\left(T^{c_{3}} T^{c_{4}}\right)_{c_{2}}^{c_{1}}[\right. & \left.b_{B_{0}\left(m_{t}^{2}+\xi \gamma ; m_{t}, 0\right)}^{R}\right|_{1 \leftrightarrow 2, m_{t} \rightarrow-m_{t}} B_{0}\left(m_{t}^{2} ; m_{t}, 0\right) \\
& \left.\left.\quad-\frac{2 m_{t}^{3}\langle 34\rangle[12]}{[34]\langle 4|2| 4]} B_{0}^{\prime}\left(m_{t}^{2} ; m_{t}, 0\right)\right]+(3 \leftrightarrow 4)\right\} .
\end{aligned}
$$

\subsection{Contributions of the counterterm diagrams}

The counterterm diagrams can be color decomposed as follows:

$$
\mathcal{M}_{\bar{t} t \rightarrow g g}^{\mathrm{ct}}=2 g^{2}\left[\left(T^{c_{3}} T^{c_{4}}\right)_{c_{2}}^{c_{1}} A^{\mathrm{ct}}\left(1_{\bar{t}}^{-}, 2_{t}^{-}, 3_{g}^{-}, 4_{g}^{-}\right)+\left(T^{c_{4}} T^{c_{3}}\right)_{c_{2}}^{c_{1}} A^{\mathrm{ct}}\left(1_{\bar{t}}^{-}, 2_{t}^{-}, 4_{g}^{-}, 3_{g}^{-}\right)\right]
$$

The color-ordered counterterm diagram $A^{\text {ct }}$ can be computed using the color-ordered Feynman rules after performing the shift (4.8). We will focus on $A^{\text {ct }}\left(1_{\bar{t}}^{-}, 2_{t}^{-}, 3_{g}^{-}, 4_{g}^{-}\right)$, since $A^{\text {ct }}\left(1_{\bar{t}}^{-}, 2_{t}^{-}, 4_{g}^{-}, 3_{g}^{-}\right)$can be obtained via the substitution $3 \leftrightarrow 4$. The corresponding color ordered counterterm diagrams are depicted in figure 9.

The diagram $D_{a}$ in figure 9 is related to the tree-level amplitudes where $1_{\bar{t}}$ is continued off-shell. These amplitudes can be derived by a recursion relation as described in appendix B.2. Using a decomposition similar to the one in eq. (2.18), the counterterm diagram $D_{a}$ can be written as $D_{a}=A^{m}+A^{\psi}+A^{k}$, whose bubble terms can be read from eq. (2.19), (2.20), and (2.21). Substituting the expansion of $J$ from (B.17) into these 
expressions, we find

$$
\begin{aligned}
& A^{m}=-\frac{g^{2} C_{F}}{8 \pi^{2}}\{ \frac{1}{\xi} B_{0}\left(m_{t}^{2} ; m_{t}, 0\right) \frac{2 m_{t}^{3}\langle 34\rangle[12]}{\langle 4|1| 4]^{2}[34]}-B_{0}\left(m_{t}^{2} ; m_{t}, 0\right) \frac{m_{t}^{2}[1|3| 2]}{\langle 4|1| 4][34]^{2}} \\
&+B_{0}\left(m_{t}^{2} ; m_{t}, 0\right) \frac{m_{t}\langle 34\rangle\left([14]\langle 4|1| 4][32]+m_{t}[14\rangle[43][42]\right)}{\langle 4|1| 4]^{2}[34]^{2}} \\
&\left.-B_{0}\left(m_{t}^{2} ; m_{t}, 0\right) \frac{m_{t}[1|4| 3 \mid 2]}{2\langle 4|1| 4][34]^{2}}\right\}, \\
& A^{\psi}=-\frac{g^{2} C_{F}}{8 \pi^{2}} B_{0}^{\prime}\left(m_{t}^{2} ; m_{t}, 0\right)\left\{\frac{2 m_{t}^{3}\langle 34\rangle[12]}{\langle 4|1| 4][34]}\right\}, \\
& A^{k}=\frac{g^{2} C_{F}}{8 \pi^{2}} B_{0}\left(m_{t}^{2} ; m_{t}, 0\right)\left\{\frac{m_{t}^{2}\langle 34\rangle\left(m_{t}[q 2][14]\langle 4 q\rangle+\langle q|1| 2][14\rangle[q 4]\right)}{\langle 4|1| 4]^{2}\langle q|1| q][34]}-\frac{m_{t}[12]\langle 34\rangle}{\langle 4|1| 4][34]}\right\} .
\end{aligned}
$$

We have kept the full $q$-dependence above, in order to demonstrate that it cancels exactly between the bubble computed from the cut and the counterterm. In practice, we might set $q=k_{4}$ throughout, in which case the $q$-dependent terms simply vanish.

The external leg counterterm diagram $D_{b}$ in figure 9 can be obtained from $D_{a}$ by performing the substitutions $1 \leftrightarrow 2,3 \leftrightarrow 4$, and $m_{t} \rightarrow-m_{t}$.

Finally, there are contributions to the coefficient of $B_{0}\left(m_{t}^{2} ; m_{t}, 0\right)$ coming from all other self-energy counterterms. In this case, there is only one, namely diagram $D_{c}$. The counterterm separates the scattering process into two off-shell currents. For the example at hand, the currents are simply the cubic interactions, and the Feynman rules give the bubble coefficient

$$
\begin{aligned}
D_{c}=\frac{g^{2} C_{F}}{8 \pi^{2}} \frac{m_{t}}{\langle 4|1| 4]^{2}[34]^{2}}[ & \left(2 m_{t}^{2}-\langle 4|1| 4]\right)([14\rangle[34]\langle 32]+[13]\langle 43\rangle[42]) \\
& \left.+2 m_{t}\langle 4|1| 4]([14\rangle[42]-[13]\langle 32])\right] .
\end{aligned}
$$

The bubble contribution of the counterterm diagrams, eq. (4.18), is then

$$
2 g^{2}\left[\left(T^{c_{3}} T^{c_{4}}\right)_{c_{2}}^{c_{1}}\left(D_{a}+D_{b}+D_{c}\right)+(3 \leftrightarrow 4)\right] .
$$

\subsection{Total bubble coefficient}

Here, we assemble all the contributions to the coefficient of $B_{0}\left(m_{t}^{2} ; m_{t}, 0\right)$. The bubble coefficient coming from the cuts and counterterms is obtained summing the contributions (4.16), (4.17), and (4.20). After the cancellation of divergent and gauge-dependent 


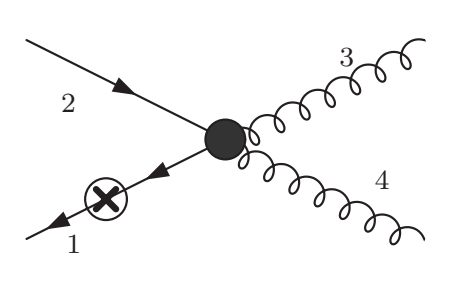

$D_{a}$

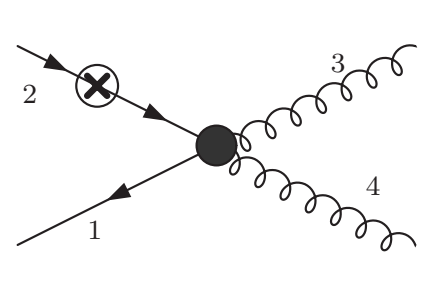

$D_{b}$

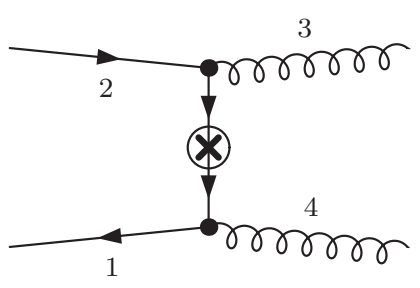

$D_{c}$

Figure 9. Counterterm diagrams contributing to the coefficient of the on-shell bubble $B_{0}\left(m_{t}^{2} ; m_{t} ; 0\right)$.

pieces between the cut-diagram and counterterm contributions, and then recovering the on-shell limit, we find the following result:

$$
\begin{aligned}
& \frac{g^{4} C_{F}}{4 \pi^{2}} \frac{m_{t}\left(T^{c_{3}} T^{c_{4}}\right)_{c_{2}}^{c_{1}}}{\langle 4|1| 4]^{2}[34]^{2}}\left[-2 m_{t}^{2}[13]\langle 34\rangle[42]+2 m\langle 4|1| 4]([13\rangle[32]+[14\rangle[42])\right. \\
& \left.-m_{t} s_{34}([13\rangle[32]+[14]\langle 42])-\langle 34\rangle\langle 4|1| 4]([13][42]-[14][32])\right]+(3 \leftrightarrow 4) .
\end{aligned}
$$

To compute the bubble coefficient for the entire amplitude, one would need the contributions from all remaining helicity amplitudes. It is easy to translate the results above to any other helicity amplitude whose gluons have equal helicities, either by overall parity conjugation of the amplitude, or by conjugating the individual massive spinors. The conventions for massive spinors (see appendix B) allow their individual conjugation except in spinor strings where they appear on both ends; these strings can be split using Schouten identities. Thus the remaining nontrivial computation for the leading-color part would be any single configuration with opposite helicities for the two gluons.

There are also coefficients multiplying the differentiated bubble, $B_{0}^{\prime}\left(m_{t}^{2} ; m_{t}, 0\right)$, arising from the various counterterms via $\delta Z_{\psi}$. They are proportional to the tree-level amplitude. The counterterm for external wavefunction renormalization cancels exactly with the cut diagram in this coefficient, as shown in section 2, but other counterterms need to be treated separately.

\section{Summary}

We close this article with a brief review of our procedure.

1. For each massive external fermion, with momentum denoted by $k$, choose the shift direction $\bar{k}$. Define the off-shell continuation $\hat{k}=k+\xi \bar{k}$, where $\xi$ parametrizes the shift, and choose another external leg to absorb the shift in order to conserve momentum, as in equation (2.1). 
2. Compute the tree-level inputs for the cuts in the shifted momentum channels. The cuts may diverge as $1 / \xi$. The expressions are needed through $\mathcal{O}\left(\xi^{0}\right)$. The expansion might be done by a contour integral, or by generating the $\mathcal{O}\left(\xi^{-1}\right)$ and $\mathcal{O}\left(\xi^{0}\right)$ terms separately.

3. The cut integrals have now been regulated, and the coefficients of master integrals $A_{0}$ and $B_{0}$ can be found in the usual way. Keep only the $\mathcal{O}\left(\xi^{0}\right)$ term. The $1 / \xi$-divergent term is ignored, unless it is desired for a consistency check. It will be proportional to the tree amplitude, as shown in equations (2.7) and (2.15). Because of the off-shell continuation, the cut depends on the gauge choice made for the cut gluon.

4. Compute the counterterms from tree-level currents. The counterterms for external leg corrections are computed at $\mathcal{O}\left(\xi^{0}\right)$. The divergent part cancels the divergence of the loop exactly and may likewise be ignored. Similarly, the coefficient of $B_{0}^{\prime}$ will cancel and may be discarded. In the spinor-helicity method, one must include the term $\mathcal{M}_{k}$ from equation (2.21), making the same gauge choice for the cut gluon as in the cut integral.

All other counterterms for internal legs and vertices are finite and can be computed from unshifted expressions. Considered separately, the counterterms depend on the gauge choices made for external legs. Gauge invariance is restored in the sum. It is the internal counterterms that provide the surviving contributions to the differentiated bubble $B_{0}^{\prime}$, via the renormalization constant $\delta Z_{\psi}$.

\section{Acknowledgments}

We are grateful to Leandro Almeida for collaborating in the early stages of this project. We thank Zvi Bern and David Kosower for insightful comments and feedback on a draft of this manuscript, and Alexander Ochirov for corrections to the first version. We acknowledge Simon Badger and Harald Ita for useful discussions. This research was supported in part by the National Science Foundation under Grant No. NSF PHY05-51164; we thank the KITP for its hospitality. R.B. is supported by the Agence Nationale de la Recherche under grant ANR-09-CEXC-009-01. E.M. is supported by the European Research Council under Advanced Investigator Grant ERC-AdG-228301.

\section{A Master integrals, double and single cuts}

The master integrals that pertain to the wavefunction renormalization diagram are the scalar bubble,

$$
B_{0}\left(k^{2} ; m, 0\right) \equiv \frac{(2 \pi \mu)^{4-D}}{i \pi^{2}} \int d^{D} \ell \frac{1}{\ell^{2}\left((k-\ell)^{2}-m^{2}\right)},
$$

and the tadpole with massive propagator,

$$
A_{0}(m) \equiv \frac{(2 \pi \mu)^{4-D}}{i \pi^{2}} \int d^{D} \ell \frac{1}{(k-\ell)^{2}-m^{2}} .
$$


These integrals are dimensionally regularized by taking the dimensionality $D=4-2 \epsilon$. The double cut of the bubble and the single cut of the tadpole are obtained by substituting the propagators with the corresponding delta functions:

$$
\begin{aligned}
\Delta_{2, k} B_{0}\left(k^{2} ; m, 0\right) & \equiv \frac{(2 \pi \mu)^{4-D}}{i \pi^{2}} \int d^{D} \ell \delta\left(\ell^{2}\right) \delta\left((k-\ell)^{2}-m^{2}\right), \\
\Delta_{1, k} A_{0}(m) & \equiv \frac{(2 \pi \mu)^{4-D}}{i \pi^{2}} \int d^{D} \ell \delta\left((k-\ell)^{2}-m^{2}\right) .
\end{aligned}
$$

The rule for computing the double cut from the left and right tree amplitudes is

$$
\Delta_{2, k} \mathcal{M}=\int d \mu_{2, k} \mathcal{M}_{L}(\ell) \mathcal{M}_{R}(\ell), \quad d \mu_{2, k} \equiv-\frac{1}{(2 \pi)^{4}} d^{D} \ell \delta\left(\ell^{2}\right) \delta\left((k-\ell)^{2}-m^{2}\right) .
$$

The rule for computing the single cut from tree amplitudes is

$$
\Delta_{1, k} \mathcal{M}=\int d \mu_{1, k} \mathcal{M}_{T}(\ell), \quad d \mu_{1, k} \equiv \frac{i}{(2 \pi)^{4}} d^{D} \ell \delta\left((k-\ell)^{2}-m^{2}\right) .
$$

For convenience, we list the reduction of several double-cut integrals,

$$
\begin{aligned}
\int d \mu_{2, k} & =-\frac{i}{16 \pi^{2}} \Delta_{2, k} B_{0}\left(k^{2} ; m, 0\right), \\
\int d \mu_{2, k}(R \cdot \ell) & =-\frac{i}{16 \pi^{2}} \frac{\left(k^{2}-m^{2}\right)}{2 k^{2}}(R \cdot k) \Delta_{2, k} B_{0}\left(k^{2} ; m, 0\right), \\
\int d \mu_{2, k} \frac{(R \cdot \ell)}{(\ell+p)^{2}-m^{2}} & =-\frac{i}{32 \pi^{2}} \frac{k^{2}(p \cdot R)-(k \cdot p)(k \cdot R)}{k^{2} p^{2}-(k \cdot p)^{2}} \Delta_{2, k} B_{0}\left(k^{2} ; m, 0\right),
\end{aligned}
$$

and single-cut integrals,

$$
\begin{aligned}
& \int d \mu_{1, k} \frac{1}{(\ell+p)^{2}}=0, \\
& \int d \mu_{1, k} \frac{(R \cdot \ell)}{(\ell+p)^{2}}=-\frac{1}{32 \pi^{2}} \frac{R \cdot(k+p)}{(k+p)^{2}} \Delta_{1, k} A_{0}(m) .
\end{aligned}
$$

Here, $R$ and $p$ are any 4 -vectors.

\section{B Massive spinors}

We use the formalism of massive spinors developed by Kleiss and Stirling [45].

For assistance in calculations, we have used the specific representation of massive spinors in terms of an arbitrary null "reference" momentum $\eta$, which is the same for all particles, as follows [68]. For an on-shell particle of mass $m$ and momentum $p$, define

$$
p^{b}=p-\frac{m^{2}}{2 p \cdot \eta} \eta
$$

which is another null vector.

Then the massive spinors are defined as

$$
\left.|p\rangle=\frac{(\not p+m) \mid \eta]}{\left[p^{b} \eta\right]}, \quad \mid p\right]=\frac{(\not p+m)|\eta\rangle}{\left\langle p^{b} \eta\right\rangle},
$$


whose conjugates are

$$
\langle p|=\frac{[\eta \mid(\not p+m)}{\left[\eta p^{b}\right]}, \quad\left[p \mid=\frac{\langle\eta|(\not p+m)}{\left\langle\eta p^{b}\right\rangle} .\right.
$$

For an antiparticle, the mass $m$ should be replaced by $-m$ everywhere. These formulas are smooth in the massless limit.

We have also used the Mathematica package S@M [72] to help with spinor manipulations and numerical evaluation.

\section{B.1 Some identities for massive spinors}

Among the identities obeyed by the massive spinors, we find the following particularly useful in our computations.

The contractions of spinors of the same chirality are antisymmetric as usual, while the mixed spinor product is symmetric:

$$
\langle i j\rangle=-\langle j i\rangle, \quad[i j]=-[j i], \quad\langle i j]=[j i\rangle .
$$

The spinor representation of a momentum vector can be replaced by the massive spinors using

$$
\begin{array}{ll}
\not p=|p\rangle[p|+| p]\langle p|-m & \text { for a particle, } \\
\not p=|p\rangle[p|+| p]\langle p|+m & \text { for an antiparticle. }
\end{array}
$$

The slash is implicit inside spinor products. Note that $\langle i|j| i]=2 p_{i} \cdot p_{j}$.

Finally, the Schouten identity is still valid for any four spinors of the same type,

$$
\langle i j\rangle\langle k l\rangle+\langle i k\rangle\langle l j\rangle+\langle i l\rangle\langle j k\rangle=0, \quad[i j][k l]+[i k][l j]+[i l][j k]=0,
$$

while of course the three-spinor uncontracted versions remain valid for any massless spinors $a, b, c$,

$$
|a\rangle\langle b c\rangle+|b\rangle\langle c a\rangle+|c\rangle\langle a b\rangle=0, \quad \mid a][b c]+\mid b][c a]+\mid c][a b]=0 .
$$

\section{B.2 Tree-level formulas}

Here we list the tree amplitudes needed for the cut in the example of section 4. They are adapted from [73]. ${ }^{5}$ The three- and five-point amplitudes, which are ingredients in the cut computation, are given in a form that allows straightforward parity conjugation of the fermions.

The convention we follow here is that gluon momenta are directed outward, while quark and anti-quark momenta are directed inward. The mass of the quark is $m$.

\footnotetext{
${ }^{5}$ Equivalent formulas were derived earlier in [74-76], also by on-shell recursion relations.
} 
Three-point amplitudes. The relevant three-point amplitudes, valid also in the offshell continuation, are

$$
\begin{aligned}
& A\left(1_{\bar{q}}^{-}, 2_{q}^{-}, 3_{g}^{-}\right)=\left[1\left|\left(\frac{\mid q]\langle 3|+| 3\rangle[q \mid}{[q 3]}\right)\right| 2\right], \\
& A\left(1_{\bar{q}}^{-}, 2_{q}^{-}, 3_{g}^{+}\right)=\left[1\left|\left(\frac{|q\rangle[3|+| 3]\langle q|}{\langle q 3\rangle}\right)\right| 2\right] .
\end{aligned}
$$

The quantity inside parentheses is the polarization vector, which depends on the null reference momentum $q$. On-shell, the amplitude is independent of $q$.

Four-point amplitudes. The tree-level amplitude corresponding to the one-loop amplitude of interest is

$$
A\left(1_{\bar{q}}^{-}, 2_{q}^{-}, 3_{g}^{-}, 4_{g}^{-}\right)=\frac{m\langle 34\rangle[12]}{\left(2 p_{2} \cdot p_{3}\right)[34]}
$$

Five-point amplitudes. The amplitudes entering the double cuts (4.9) and (4.12) are

$$
\begin{aligned}
A\left(1_{\bar{q}}^{+}, 2_{q}^{-}, 3_{g}^{-}, 4_{g}^{-}, 5_{g}^{-}\right)= & \frac{m\left\langle 3\left|p_{45}\right| 1 \mid 5\right\rangle(\langle 14][32]-\langle 13][42])}{\left(2 p_{1} \cdot p_{5}\right)\left(2 p_{2} \cdot p_{3}\right)[34]^{2}[45]} \\
A\left(1_{\bar{q}}^{+}, 2_{q}^{-}, 3_{g}^{-}, 4_{g}^{-}, 5_{g}^{+}\right)= & \frac{\left[5|1| p_{34}|2| 3\right\rangle\left([25]\left\langle 1\left|p_{34}\right| 2 \mid 3\right\rangle-\left[2\left|p_{34}\right| d_{51}|3\rangle\langle 15]\right)\right.}{\left(2 p_{1} \cdot p_{5}\right)\left(2 p_{2} \cdot p_{3}\right)[34][45]\left\langle 5\left|p_{34}\right| 2 \mid 3\right\rangle} \\
& -\frac{m\langle 43\rangle^{3}\left(\left\langle 1\left|p_{34}\right| 5\right\rangle\left\langle 3\left|p_{45}\right| 2\right]-\left\langle 1\left|p_{45}\right| 3\right\rangle\left\langle 5\left|p_{34}\right| 2\right]\right)}{\left(p_{1}+p_{2}\right)^{4}\langle 45\rangle\langle 35\rangle\left\langle 5\left|p_{34}\right| 2 \mid 3\right\rangle} \\
A\left(1_{\bar{q}}^{+}, 2_{q}^{-}, 3_{g}^{-}, 4_{g}^{+}, 5_{g}^{-}\right)= & \frac{m\langle 35\rangle^{3}\left(\left\langle 1\left|p_{34}\right| 5\right\rangle\left\langle 3\left|p_{45}\right| 2\right]-\left\langle 1\left|p_{45}\right| 3\right\rangle\left\langle 5\left|p_{34}\right| 2\right]\right)}{\left(p_{1}+p_{2}\right)^{4}\langle 34\rangle\langle 45\rangle\left\langle 5\left|p_{34}\right| 2 \mid 3\right\rangle} \\
& +\frac{[4|1| 5\rangle[4|2| 3\rangle}{\left(2 p_{1} \cdot p_{5}\right)\left(2 p_{2} \cdot p_{3}\right)[34][45]\left\langle 5\left|p_{34}\right| 2 \mid 3\right\rangle}(m\langle 14]\langle 53\rangle[42] \\
A\left(1_{\bar{q}}^{+}, 2_{q}^{-}, 3_{g}^{+}, 4_{g}^{-}, 5_{g}^{-}\right)= & -\frac{\left.\left[3|2| p_{45}|1| 5\right\rangle\left(\langle 13]\left\langle 5|1| p_{45}\right| 2\right]+\left\langle 1\left|p_{45}\right| d_{32} \mid 5\right\rangle[32]\right)}{\left(2 p_{1} \cdot p_{5}\right)\left(2 p_{2} \cdot p_{3}\right)[34][45]\left\langle 5|1| p_{45} \mid 3\right\rangle} \\
& +\frac{m\langle 45\rangle^{3}\left(\left\langle 1\left|p_{34}\right| 5\right\rangle\left\langle 3\left|p_{45}\right| 2\right]-\left\langle 1\left|p_{45}\right| 3\right\rangle\left\langle 5\left|p_{34}\right| 2\right]\right)}{\left(p_{1}+p_{2}\right)^{4}\langle 34\rangle\langle 35\rangle\left\langle 5|1| p_{45} \mid 3\right\rangle}
\end{aligned}
$$

where $p_{i j} \equiv p_{i}+p_{j}$ and $d_{i j} \equiv p_{i}-p_{j}$. Similar amplitudes with the opposite helicity choice for $1_{\bar{q}}$ are given by exchanging angle brackets $|1\rangle,\langle 1|$ with square brackets $\left.\mid 1\right],[1 \mid$. (The quark $2_{q}$ can be conjugated similarly, but we do not need that in the present paper.) This type of conjugation is valid as long as the derivation of the tree amplitudes did not apply any identities such as the Schouten identities, (B.7), which does not respect conjugation of a single massive spinor if it is contracted with another. 
Off-shell four-point currents. For the counterterm, we use a more general formula for the tree-level four-point current. This expression is valid when the particle $1_{\bar{q}}$ is continued off shell and its external spinor is stripped off. Because it is off shell, it depends on the reference momenta $q_{3}$ and $q_{4}$ of gluons 3 and 4 , respectively. The full expression is

$$
\begin{aligned}
J_{q_{3}, q_{4}}\left(1_{\bar{q}}^{-}, 2_{q}^{-}, 3_{g}^{-}, 4_{g}^{-}\right)= & \frac{1}{\left[q_{3} 3\right]\left[q_{4} 4\right]}\left[\frac { 1 } { \langle 3 | 2 | 3 ] } \left(\left[1 q_{4}\right]\left\langle 4|1| q_{3}\right]\langle 32]-[14\rangle\left[q_{4}|2| 3\right\rangle\left[q_{3} 2\right]\right.\right. \\
& \left.-m\left[1 q_{4}\right]\langle 43\rangle\left[q_{3} 2\right]-m[14\rangle\left[q_{4} q_{3}\right]\langle 32]\right) \\
& +\frac{1}{[34]}\left(\left[1 q_{4}\right]\langle 42]\left[q_{3} 4\right]+[14\rangle\left[q_{4} 2\right]\left[q_{3} 4\right]\right. \\
& \left.\left.+\left[1 q_{3}\right]\langle 32]\left[q_{4} 3\right]+[13\rangle\left[q_{3} 2\right]\left[q_{4} 3\right]+\frac{\left[1\left|d_{34}\right| 2\right]\left[q_{3} q_{4}\right]}{2}\right)\right] .
\end{aligned}
$$

For the example discussed in section 4, we need the current for the process where only $k_{1}$ is off shell, and we choose $q_{3}=k_{4}, q_{4}=k_{3}$ throughout. This current, with the spinor [1 stripped off, is given by

$$
J=\frac{\left.\left.\left.p_{34}|2| 3\right\rangle[32]+m \mid 3\right]\langle 43\rangle[42]+\left(m^{2}-p_{1}^{2}\right) \mid 3\right][23\rangle}{2 p_{2} \cdot p_{3}[34]^{2}}-\frac{\left.p_{34} \mid 2\right]}{2[34]^{2}} .
$$

Open Access. This article is distributed under the terms of the Creative Commons Attribution Noncommercial License which permits any noncommercial use, distribution, and reproduction in any medium, provided the original author(s) and source are credited.

\section{References}

[1] C. Buttar, S. Dittmaier, V. Drollinger, S. Frixione, A. Nikitenko, et al., Les Houches physics at TeV colliders 2005, standard model and Higgs working group: summary report, hep-ph/0604120 [INSPIRE].

[2] NLO Multileg Working Group collaboration, Z. Bern et al., The NLO multileg working group: summary report, arXiv:0803.0494 [INSPIRE].

[3] R. Cutkosky, Singularities and discontinuities of Feynman amplitudes, J. Math. Phys. 1 (1960) 429 [INSPIRE].

[4] W. van Neerven, Dimensional regularization of mass and infrared singularities in two loop on-shell vertex functions, Nucl. Phys. B 268 (1986) 453 [INSPIRE].

[5] Z. Bern, L.J. Dixon, D.C. Dunbar and D.A. Kosower, One loop $N$ point gauge theory amplitudes, unitarity and collinear limits, Nucl. Phys. B 425 (1994) 217 [hep-ph/9403226] [INSPIRE].

[6] Z. Bern, L.J. Dixon, D.C. Dunbar and D.A. Kosower, Fusing gauge theory tree amplitudes into loop amplitudes, Nucl. Phys. B 435 (1995) 59 [hep-ph/9409265] [INSPIRE].

[7] Z. Bern, L.J. Dixon and D.A. Kosower, One loop amplitudes for $e^{+} e^{-}$to four partons, Nucl. Phys. B 513 (1998) 3 [hep-ph/9708239] [InSPIRE]. 
[8] Z. Bern, V. Del Duca, L.J. Dixon and D.A. Kosower, All non-maximally-helicity-violating one-loop seven-gluon amplitudes in $N=4$ super-Yang-Mills theory, Phys. Rev. D 71 (2005) 045006 [hep-th/0410224] [INSPIRE].

[9] R. Britto, F. Cachazo and B. Feng, Generalized unitarity and one-loop amplitudes in $N=4$ super-Yang-Mills, Nucl. Phys. B 725 (2005) 275 [hep-th/0412103] [INSPIRE].

[10] P. Mastrolia, On triple-cut of scattering amplitudes, Phys. Lett. B 644 (2007) 272 [hep-th/0611091] [INSPIRE].

[11] D. Forde, Direct extraction of one-loop integral coefficients, Phys. Rev. D 75 (2007) 125019 [arXiv: 0704.1835] [INSPIRE].

[12] G. Ossola, C.G. Papadopoulos and R. Pittau, Reducing full one-loop amplitudes to scalar integrals at the integrand level, Nucl. Phys. B 763 (2007) 147 [hep-ph/0609007] [inSPIRE].

[13] R. Ellis, W. Giele and Z. Kunszt, A numerical unitarity formalism for evaluating one-loop amplitudes, JHEP 03 (2008) 003 [arXiv: 0708.2398] [INSPIRE].

[14] W.B. Kilgore, One-loop integral coefficients from generalized unitarity, arXiv:0711.5015 [INSPIRE].

[15] W.T. Giele, Z. Kunszt and K. Melnikov, Full one-loop amplitudes from tree amplitudes, JHEP 04 (2008) 049 [arXiv:0801.2237] [INSPIRE].

[16] C. Berger, Z. Bern, L. Dixon, F. Febres Cordero, D. Forde, et al., An automated implementation of on-shell methods for one-loop amplitudes, Phys. Rev. D 78 (2008) 036003 [arXiv:0803.4180] [INSPIRE].

[17] S. Badger, Direct extraction of one loop rational terms, JHEP 01 (2009) 049 [arXiv:0806.4600] [INSPIRE].

[18] R. Ellis, W.T. Giele, Z. Kunszt and K. Melnikov, Masses, fermions and generalized d-dimensional unitarity, Nucl. Phys. B 822 (2009) 270 [arXiv:0806.3467] [INSPIRE].

[19] C.F. Berger and D. Forde, Multi-parton scattering amplitudes via on-shell methods, Ann. Rev. Nucl. Part. Sci. 60 (2010) 181 [arXiv:0912.3534] [InSPIRE].

[20] R. Britto, Loop amplitudes in gauge theories: modern analytic approaches, J. Phys. A 44 (2011) 454006 [arXiv: 1012.4493] [InSPIRE].

[21] R. Ellis, Z. Kunszt, K. Melnikov and G. Zanderighi, One-loop calculations in quantum field theory: from Feynman diagrams to unitarity cuts, arXiv:1105.4319 [INSPIRE].

[22] L. Brown and R. Feynman, Radiative corrections to Compton scattering, Phys. Rev. 85 (1952) 231 [INSPIRE].

[23] D. Melrose, Reduction of Feynman diagrams, Nuovo Cim. 40 (1965) 181 [INSPIRE].

[24] G. Passarino and M. Veltman, One loop corrections for $e^{+} e^{-}$annihilation into $\mu^{+} \mu^{-}$in the Weinberg model, Nucl. Phys. B 160 (1979) 151 [InSPIRE].

[25] G. 't Hooft and M. Veltman, Scalar one loop integrals, Nucl. Phys. B 153 (1979) 365 [INSPIRE].

[26] W. van Neerven and J. Vermaseren, Large loop integrals, Phys. Lett. B 137 (1984) 241 [INSPIRE].

[27] R.G. Stuart, Algebraic reduction of one loop Feynman diagrams to scalar integrals, Comput. Phys. Commun. 48 (1988) 367 [INSPIRE]. 
[28] R.G. Stuart and A. Gongora, Algebraic reduction of one loop Feynman diagrams to scalar integrals. 2., Comput. Phys. Commun. 56 (1990) 337 [INSPIRE].

[29] G. van Oldenborgh and J. Vermaseren, New algorithms for one loop integrals, Z. Phys. C 46 (1990) 425 [INSPIRE].

[30] Z. Bern, L.J. Dixon and D.A. Kosower, Dimensionally regulated one loop integrals, Phys. Lett. B 302 (1993) 299 [Erratum ibid. B 318 (1993) 649] [hep-ph/9212308] [INSPIRE].

[31] Z. Bern, L.J. Dixon and D.A. Kosower, Dimensionally regulated pentagon integrals, Nucl. Phys. B 412 (1994) 751 [hep-ph/9306240] [INSPIRE].

[32] J. Fleischer, F. Jegerlehner and O. Tarasov, Algebraic reduction of one loop Feynman graph amplitudes, Nucl. Phys. B 566 (2000) 423 [hep-ph/9907327] [INSPIRE].

[33] T. Binoth, J. Guillet and G. Heinrich, Reduction formalism for dimensionally regulated one loop $N$ point integrals, Nucl. Phys. B 572 (2000) 361 [hep-ph/9911342] [INSPIRE].

[34] A. Denner and S. Dittmaier, Reduction of one loop tensor five point integrals, Nucl. Phys. B 658 (2003) 175 [hep-ph/0212259] [INSPIRE].

[35] G. Duplancic and B. Nizic, Reduction method for dimensionally regulated one loop $N$ point Feynman integrals, Eur. Phys. J. C 35 (2004) 105 [hep-ph/0303184] [INSPIRE].

[36] A. Denner and S. Dittmaier, Reduction schemes for one-loop tensor integrals, Nucl. Phys. B 734 (2006) 62 [hep-ph/0509141] [INSPIRE].

[37] R. Ellis and G. Zanderighi, Scalar one-loop integrals for QCD, JHEP 02 (2008) 002 [arXiv: 0712.1851] [INSPIRE].

[38] P. Mastrolia, G. Ossola, T. Reiter and F. Tramontano, Scattering amplitudes from unitarity-based reduction algorithm at the integrand-level, JHEP 08 (2010) 080 [arXiv: 1006.0710] [INSPIRE].

[39] Z. Bern and A. Morgan, Massive loop amplitudes from unitarity, Nucl. Phys. B 467 (1996) 479 [hep-ph/9511336] [InSPIRE].

[40] S. Badger, Generalised unitarity at one-loop with massive fermions, Nucl. Phys. Proc. Suppl. 183 (2008) 220 [arXiv:0807.1245] [INSPIRE].

[41] R. Britto and B. Feng, Solving for tadpole coefficients in one-loop amplitudes, Phys. Lett. B 681 (2009) 376 [arXiv:0904.2766] [INSPIRE].

[42] R. Britto and E. Mirabella, Single cut integration, JHEP 01 (2011) 135 [arXiv:1011.2344] [INSPIRE].

[43] F.A. Berends, R. Kleiss, P. De Causmaecker, R. Gastmans and T.T. Wu, Single Bremsstrahlung processes in gauge theories, Phys. Lett. B 103 (1981) 124 [INSPIRE].

[44] P. De Causmaecker, R. Gastmans, W. Troost and T.T. Wu, Multiple Bremsstrahlung in gauge theories at high-energies. 1. General formalism for quantum electrodynamics, Nucl. Phys. B 206 (1982) 53 [inSPIRE].

[45] R. Kleiss and W. Stirling, Spinor techniques for calculating $p \bar{p} \rightarrow W^{+-} / Z_{0}+$ jets, Nucl. Phys. B 262 (1985) 235 [inSPIRE].

[46] Z. Xu, D.-H. Zhang and L. Chang, Helicity amplitudes for multiple Bremsstrahlung in massless nonabelian gauge theories, Nucl. Phys. B 291 (1987) 392 [INSPIRE]. 
[47] J. Gunion and Z. Kunszt, Improved analytic techniques for tree graph calculations and the gg $q \bar{q}$ lepton anti-lepton subprocess, Phys. Lett. B 161 (1985) 333 [INSPIRE].

[48] E. Braaten and J. Leveille, Higgs boson decay and the running mass, Phys. Rev. D 22 (1980) 715 [INSPIRE].

[49] N. Sakai, Perturbative QCD corrections to the hadronic decay width of the Higgs boson, Phys. Rev. D 22 (1980) 2220 [inSPIRE].

[50] T. Inami and T. Kubota, Renormalization group estimate of the hadronic decay width of the Higgs boson, Nucl. Phys. B 179 (1981) 171 [InSPIRE].

[51] M. Drees and K.-i. Hikasa, Note on QCD corrections to hadronic Higgs decay, Phys. Lett. B 240 (1990) 455 [Erratum ibid. B 262 (1991) 497] [INSPIRE].

[52] T. Hahn, Generating Feynman diagrams and amplitudes with FeynArts 3, Comput. Phys. Commun. 140 (2001) 418 [hep-ph/0012260] [INSPIRE].

[53] T. Hahn and M. Pérez-Victoria, Automatized one loop calculations in four-dimensions and d-dimensions, Comput. Phys. Commun. 118 (1999) 153 [hep-ph/9807565] [INSPIRE].

[54] T. Hahn and M. Rauch, News from FormCalc and LoopTools, Nucl. Phys. Proc. Suppl. 157 (2006) 236 [hep-ph/0601248] [inSPIRE].

[55] R. Mertig, M. Böhm and A. Denner, FEYN CALC: computer algebraic calculation of Feynman amplitudes, Comput. Phys. Commun. 64 (1991) 345 [INSPIRE].

[56] P. Nason, S. Dawson and R. Ellis, The total cross-section for the production of heavy quarks in hadronic collisions, Nucl. Phys. B 303 (1988) 607 [InSPIRE].

[57] P. Nason, S. Dawson and R. Ellis, The one particle inclusive differential cross-section for heavy quark production in hadronic collisions, Nucl. Phys. B 327 (1989) 49 [Erratum ibid. B 335 (1990) 260] [INSPIRE].

[58] W. Beenakker, H. Kuijf, W. van Neerven and J. Smith, QCD corrections to heavy quark

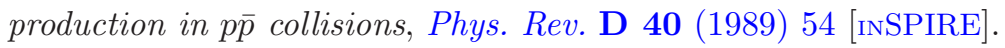

[59] W. Beenakker, W. van Neerven, R. Meng, G. Schuler and J. Smith, QCD corrections to heavy quark production in hadron hadron collisions, Nucl. Phys. B 351 (1991) 507 [INSPIRE].

[60] J.M. Campbell and R. Ellis, Radiative corrections to Z $b \bar{b}$ production, Phys. Rev. D 62 (2000) 114012 [hep-ph/0006304] [INSPIRE].

[61] S. Frixione, P. Nason and B.R. Webber, Matching NLO QCD and parton showers in heavy flavor production, JHEP 08 (2003) 007 [hep-ph/0305252] [INSPIRE].

[62] R. Britto, E. Buchbinder, F. Cachazo and B. Feng, One-loop amplitudes of gluons in SQCD, Phys. Rev. D 72 (2005) 065012 [hep-ph/0503132] [INSPIRE].

[63] R. Britto and B. Feng, Unitarity cuts with massive propagators and algebraic expressions for coefficients, Phys. Rev. D 75 (2007) 105006 [hep-ph/0612089] [INSPIRE].

[64] R. Britto, B. Feng and P. Mastrolia, The cut-constructible part of QCD amplitudes, Phys. Rev. D 73 (2006) 105004 [hep-ph/0602178] [INSPIRE].

[65] R. Britto and B. Feng, Integral coefficients for one-loop amplitudes, JHEP 02 (2008) 095 [arXiv: 0711.4284] [INSPIRE].

[66] R. Britto, B. Feng and P. Mastrolia, Closed-Form decomposition of one-loop massive amplitudes, Phys. Rev. D 78 (2008) 025031 [arXiv: 0803.1989] [InSPIRE]. 
[67] P. Mastrolia, Double-Cut of scattering amplitudes and stokes' theorem, Phys. Lett. B 678 (2009) 246 [arXiv:0905.2909] [INSPIRE].

[68] C. Schwinn and S. Weinzierl, Scalar diagrammatic rules for Born amplitudes in QCD, JHEP 05 (2005) 006 [hep-th/0503015] [INSPIRE].

[69] Z. Bern, L.J. Dixon and D.A. Kosower, One loop corrections to two quark three gluon amplitudes, Nucl. Phys. B 437 (1995) 259 [hep-ph/9409393] [INSPIRE].

[70] S. Badger, R. Sattler and V. Yundin, One-loop helicity amplitudes for t $\bar{t}$ production at hadron colliders, Phys. Rev. D 83 (2011) 074020 [arXiv:1101.5947] [INSPIRE].

[71] L.J. Dixon, Calculating scattering amplitudes efficiently, hep-ph/9601359 [INSPIRE].

[72] D. Maitre and P. Mastrolia, S@M, a Mathematica implementation of the spinor-helicity formalism, Comput. Phys. Commun. 179 (2008) 501 [arXiv:0710.5559] [inSPIRE].

[73] A. Hall, Massive quark-gluon scattering amplitudes at tree level, Phys. Rev. D 77 (2008) 025011 [arXiv:0710.1300] [INSPIRE].

[74] K. Ozeren and W. Stirling, Scattering amplitudes with massive fermions using BCFW recursion, Eur. Phys. J. C 48 (2006) 159 [hep-ph/0603071] [INSPIRE].

[75] C. Schwinn and S. Weinzierl, SUSY Ward identities for multi-gluon helicity amplitudes with massive quarks, JHEP 03 (2006) 030 [hep-th/0602012] [INSPIRE].

[76] C. Schwinn and S. Weinzierl, On-shell recursion relations for all Born QCD amplitudes, JHEP 04 (2007) 072 [hep-ph/0703021] [INSPIRE].

[77] B. Feng and Z. Zhang, Boundary contributions using fermion pair deformation, arXiv:1109.1887 [INSPIRE]. 